\title{
Elastic versus acoustic inversion for marine surveys
}

\author{
Peter Mora ${ }^{1}$, Zedong $\mathrm{Wu}^{2}$ \\ ${ }^{1}$ Maths Capital Management, 20 Kent Avenue, Warradale,Adelaide, 5046, SA, Australia \\ ${ }^{2}$ King Abdullah University of Science and Technology (KAUST), Thuwal, 23955-6900, Saudi Arabia
}

\section{SUMMARY}

Full Wavefield Inversion (FWI) is a powerful and elegant approach for seismic imaging that is on the way to becoming the method of choice when processing exploration or global seismic data. In the case of processing marine survey data, one may be tempted to assume acoustic FWI is sufficient given that only pressure waves exist in the water layer. In this paper, we pose the question as to whether or not in theory - at least for a hard water bottom case - it should be possible to resolve the shear modulus or S-wave velocity in a marine setting using large offset data. We therefore conduct numerical experiments with idealized marine data calculated with the elastic wave equation. We study two cases, FWI of data due to a diffractor model, and FWI of data due to a fault model. We find that at least in idealized situation, elastic FWI of hard waterbottom data is capable of resolving between the two Lamé parameters $\lambda$ and $\mu$. Another numerical experiment with a soft waterbottom layer gives the same result. In contrast, acoustic FWI of the synthetic elastic data results in a single image of the first Lamé parameter $\lambda$ which contains severe artefacts for diffraction data and noticable artefacts for layer reflection data. Based on these results, it would appear that at least, inversions of large offset marine data should be fully elastic rather than acoustic unless it has been demonstrated that for the specific case in question (offsets, model and water depth, practical issues such as soft sediment attenuation of shear waves or computational time), that an acoustic 
only inversion provides a reasonably good quality of image comparable to that of an elastic inversion. Further research with real data is required to determine the degree to which practical issues such as shear wave attenuation in soft sediments may affect this result.

Key words: Full waveform inversion, marine data inversion.

\section{INTRODUCTION}

Full Wavefield Inversion in the acoustic approximation was first proposed in the early 1980's (Lailly 1983; Tarantola 1984). During the late 1980's, Full Wavefield Inversion for the fully elastic case was developed and demonstrated (Mora 1987b; Tarantola 1988) but has not been commonly applied to exploration data due to significantly higher computational cost.

Much of the research is done in the acoustic approximation to decrease computational cost, or based on the assumption that most of the energy is in the compressional waves, particularly in the case of marine data where the waves must convert to pressure waves in the water layer. However, if one does decide to perform the inversion in the acoustic approximation, one must pose the question as to what are the consequences of this approximation. Clearly, the earth is elastic to first order, so both compressional and shear waves exist in the solid layers. We may wonder whether the acoustic approximation is appropriate. What is the consequence on the quality of the image of using the acoustic approximation, at least under ideal circumstances? And what extra information can be gained by using the elastic wave equation?

A number of papers have been published that aimed to study the consequences of applying acoustic wavefield inversion to elastic data on the P-wave velocity result (Barnes \& Charara 2009; Vigh et al. 2009; Marelli et al. 2012; He \& Plessix 2016). These papers showed inversion examples of 2D and 3D models and noted that artefacts exist on the $\mathrm{P}$-wave velocity inversion result when acoustic inversion is carried out using elastic data. Several authors have proposed methods to help mitigate the artefacts on the P-wave image that resulted from applying acoustic inversion to elastic data (Agudo et al. 2016; Albertin et al. 2016; Willemsen et al. 2016). Work towards the goal of mitigating artefacts of using the acoustic approximation includes research that improves amplitude versus incidence angle and use of multiple propagations of acoustic waves of by adjusting the density (Mulder \& Plessix 2008; Chapman et al. 2014; Hobro et al. 2014). Such research is focussed on the artefacts resulting from incorrect amplitude versus incidence angle of the $P-P$ reflections, rather than artefacts due to the acoustic inversion attempting to fit mode converted arrivals. Our goal is not the mitigate the effects 
of artefacts. Rather, we aim to study the nature of all artefacts, particularly those related to fitting mode converted energy, and to demonstrate what extra information can be gained by fully elastic inversion, for the case of large offset (incidence angle) data when mode conversions become significant. We do not study the small offset case where mode conversions can be neglected to first order and the acoustic approximation is expected to be adequate. Based on the work of (Shipp \& Singh 2002) in which 2D elastic Full Wavefield Inversion was applied to wide aperture marine seismic data to invert for the P-wave velocity, mode conversions play an important role in improving the P-wave velocity image, even for marine data.

The goals of this paper are: (1) to conduct numerical experiments for "hard waterbottom" and "soft waterbottom" models to enable the quality of Full elastic Wavefield Inversion results to be compared to Full acoustic Wavefield Inversion results of elastic data, and (2) to determine whether elastic inversion can resolve a second image (eg. the S-wave velocity or shear modulus) compared to acoustic inversion which is only capable of resolving a P-wave velocity image (or P-wave modulus). We limit this study to the case of a simulated marine survey and hence, the uppermost layer is acoustic and can support only pressure waves. This case was chosen because some researchers have argued that the lack of shear waves in the water layer justifies the application of the acoustic approach.

The following numerical experiments involve Full Wavefield Inversion (FWI) in the elastic case, and in the acoustic approximation. We apply the classical time domain FWI formulation described in (Tarantola 1984, 1988; Mora 1987b) and use a relatively broad-band source wavelet (a first derivative of a Gaussian). As such, the inversion results presented are Full Wavefield Inversions in which both the amplitudes and phases of the recorded wavefields must be matched. We assume that a smooth background velocity field is known, and that this is provided as a starting model so we will not be considering issues of cycle-skipping which can occur if the smooth initial model is not close enough to the true model. The numerical experiments aim to investigate the assumption that acoustic inversion provides adequate results in the case of large offset data, in which offsets are comparable to or larger than the depths of interest. Namely, data in which mode conversions will be present. We do not try to mitigate the effects of using acoustic inversion. We rather focus on inversions using a simple diffractors model, and a simple anticline and thrust fault model to study acoustic inversion relative to elastic inversion. Hence, our goal is to clarify, for simple and easy to understand models, the kind of artefacts that may occur when applying Full Wavefield Inversion in the acoustic approximation, and whether elastic Full Wavefield Inversion is able to resolve added information such as on the shear wave modulus. 


\section{FORWARD MODELLING AND INVERSION METHODOLOGY}

In the following, we utilize a staggered finite-dfference grid formulation to solve the elastic wave equation as described by Igel et al. (1995). This method is very briefly reviewed in Appendix A for completeness.

The Full Wavefield Inversion method used in this paper is the time domain formulation as described by Tarantola (1988) and Mora (1987b). Again, the basic equations and the conjugate gradient algorithm used in this paper are briefly reviewed in Appendix B for completeness. The inversion parameters in this paper are $\lambda, \mu$, and $\rho$. A careful choice of parameters (He et al. 2018) which considers radiation patterns can potentially do an even better job. However, such considerations are outside the scope of this paper.

\subsection{Conversion of model units to physical units}

In this paper, we have chosen dimensionless units (ie. scaled to $\Delta x=\Delta z=1$ ). To see what size of model the water layer model would correspond to, we note that simulations were done at about 8 grid-points per fundamental wavelength in the water layer. Hence, the conversion to physical uints can be achieved as follows. For a model that is $500 \times 200$ units, this equates to a model that is $500 / 8 \times 200 / 8=62.5 \times 25$ wavelengths. For $V_{p}($ water $)=1500 \mathrm{~m} / \mathrm{s}$ and a source at $10-30 \mathrm{~Hz}$ with a fundamental frequency of $20 \mathrm{~Hz}$, we have a fundamental wavelength of $1500 / 20=75$ metres, so the model size is $75 \times 62.5$ metres in $x$, and $25 \times 75$ metres in $z$, namely, $4687 \times 1875 \mathrm{~m}^{2}$. Hence, the $500 \times 200$ model is roughly $5 \mathrm{~km}$ by $2 \mathrm{~km}$ in size at 20 Hz. All the examples in this paper can be similarly scaled. Specifically, at 20 hertz, the grid spacing is approximately $\Delta x=\Delta z=75 / 8 \sim 10$ metres, and hence, a model size of $n x \times n z$ is $n x \times 10$ metres by $n z \times 10$ metres in size.

The calculations were done with a value of $\Delta t^{*}=0.175$ where here, the superscript $*$ implies model units. We can convert from the model time units to time as follows. In the water layer, we have $\lambda^{*}=1$ and $\rho^{*}=1$ so we have a model velocity of $V_{p}^{*}=\sqrt{\lambda^{*} / \rho^{*}}=1$. So for the 20 Hertz example above where we have $\Delta x=10 \mathrm{~m}$ and $V_{p}($ water $)=1500 \mathrm{~m} / \mathrm{s}$, we can write

$$
V_{p} \frac{\Delta t}{\Delta x}=1500 \frac{\Delta t}{10}=150 \Delta t=V_{p}^{*} \frac{\Delta t^{*}}{\Delta_{x}^{*}}=0.175,
$$

and hence, the time step in seconds, $\Delta t$, is given by

$$
\Delta t=0.175 / 150=0.0011667 \text { secs } .
$$


Hence, in our example for modelling a $20 \mathrm{~Hz}$ fundamental frequency, we have a grid spacing of $\Delta x=10 \mathrm{~m}$ and a time step $\Delta t=0.0011667 \mathrm{~s}$. In this case, a time axis that is 1500 points long is equivalent to a time axis of $1500 \times 0.0011667 \approx 1.75$ secs. Therefore, in all the following plots which are labeled in time steps and grid spacing, a simple conversion of model units to physical units, for a $20 \mathrm{~Hz}$ fundamental frequency, is to multiply the $x$ and $z$ ordinates by $10 \mathrm{~m}$, and to multiply the time axis by 0.0011667 secs. Thus, time axes that are respectively 1200 and 1500 model time units long are equivalent to time axes that are $1.4 \mathrm{~s}$ and $1.75 \mathrm{~s}$ long.

\subsection{Simulation of a water layer model}

Figure 1 shows the model consisting of a water layer above two solid layers. And Figure 2 shows snapshots of a simulation of a pressure source located centrally at the top of the model. The simulation was made using the staggered grid finite-difference scheme of Igel et al. (1995) to solve Equations (A.5) through (A.7). These snapshots show only pressure waves in the uppermost acoustic water layer, and significant mode converted energy in the solid layers below the water bottom. For example, one observes the $P-P$ and $P-S$ mode conversion when the pressure wave enters the solid layer at a depth of 60 units (Figure 2 (b)), and one observes the $P-P, P-S, S-P$ and $S-S$ reflected and transmitted waves when the waves intersect the halfspace boundary at a depth of 120 units (Figure 2 (c)). This illustrates the ability of the staggered finite-difference scheme to model a water layer above solid layers.

\section{DIFFRACTORS MODEL}

In the following simulations, we define a model that is $256 \times 174$ gridpoints in size, with the lower part of the model below 128 gridpoints deep being an absorbing band. Hence, the domain of the model spans from $x_{\text {left }}=0$ through to $x_{\text {right }}=255 \Delta x=255$. We define three sets of four diffractors in this model for $\rho, \lambda$ and $\mu$ located respectively at $x_{\rho}=x_{\text {left }}+$ $0.3\left(x_{\text {right }}-x_{\text {left }}\right)=76.5 \Delta x, x_{\lambda}=x_{\text {left }}+0.5\left(x_{\text {right }}-x_{\text {left }}\right)=128 \Delta x$, and at $x_{\mu}=x_{\text {left }}+$ $0.7\left(x_{\text {right }}-x_{\text {left }}\right)=178.5 \Delta x$ as shown in Figure 3, where each set of diffractors is located at four depths (40,60, 80 and 100 gridpoints deep). The model has a narrow water layer that is 10 grid spacings deep, and the model is 128 gridpoints deep before an absorbing layer is specified. In contrast, the horizontal extent of the model is 256 gridpoints wide and an absorbing band that is 30 gridpoints wide is specified on either side of this model. As such, for a source that is located centrally, the maximum offset is 128 gridpoints wide less the absorbing layer (30 gridpoints) $=98$ gridpoints. Hence, we have offset to depth ratios 


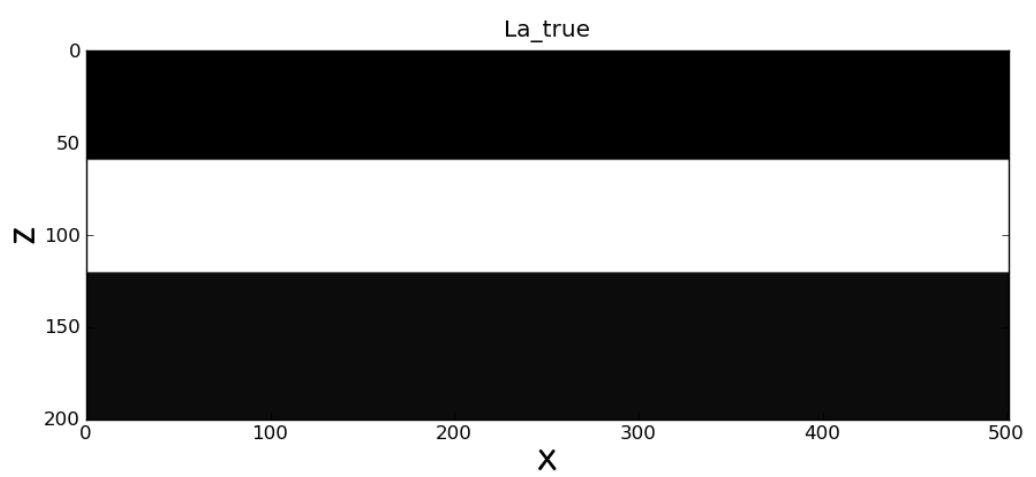

Figure 1. A 2D layer model. The uppermost layer represents water and has properties $\lambda_{w}=1, \mu_{w}=0$, and $\rho_{w}=1$. The middle layer has properties $\lambda=\mu=\rho=1$. The lower halfspace has properties $\lambda=0.1$, $\mu=0.5$, and $\rho=1$. Hence, the $P$-wave velocities of the different layers, given by $v_{p}=\sqrt{\lambda+2 \mu / \rho}$, are repectively 1 for the water layer, $\sqrt{3}$ for the solid layer below the water bottom, and $\sqrt{1.1} \approx 1.05$ for the lower halfspace. Note that the $S$-wave velocity in the middle solid layer is equal to unity (ie. it has the same speed as the $P$-wave in water).

spanning $(128-30) / 40=2.45$ through to $(128-30) / 100 \approx 1$. These can be considered as moderate to large offsets relative to depth, a realistic assumption for typical seismic surveys.

\subsection{Elastic inversion of diffractors - single shot}

In this example, a single shot record with the pressure source located centrally at the surface of the model is inverted. The residuals after 100 iterations were small and hence, the inversion was stopped after 100 iterations. The inversion results after 100 iterations are shown in Figure 4 . These indicate that the two Lamé parameters $\lambda$ and $\mu$ are well resolved from one another for this example which involves moderate to large offsets relative to the depths of the diffractors (offset to depth ratios of around 2.5:1 for the shallow diffractors, and 1:1 for the deep diffractors). Namely, one can see clearly the four diffractors in $\lambda$ at $x_{\lambda}$ in Figure 4 and four diffractors in $\mu$ at $x_{\mu}$ with only minor artefacts elsewhere. Similarly, the inversion result for density $\rho$ has four strong perturbations at the correct locations of the density diffractors $x_{\rho}$, and also smaller ghost perturbations at the locations of the diffractors in $\lambda$ and $\mu$. This indicates an imperfect resolvability between density and the Lamé parameter, and a relatively good resolvability between the two Lamé parameters $\lambda$ and $\mu$. 


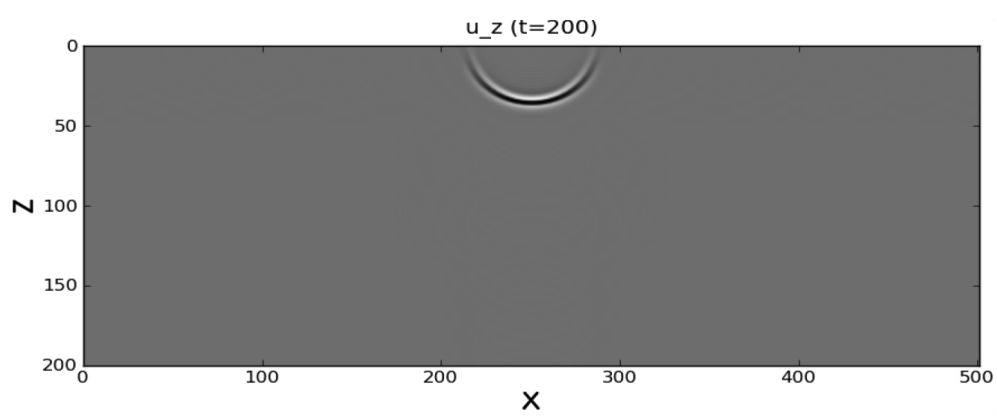

(a)

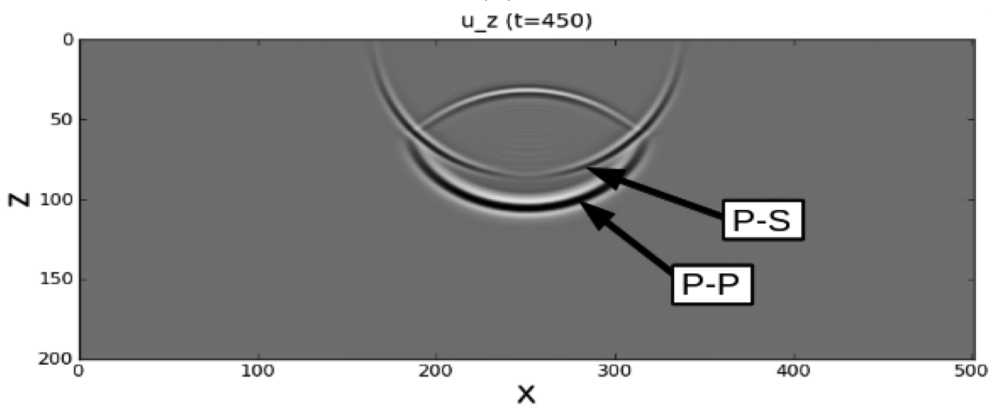

(b)

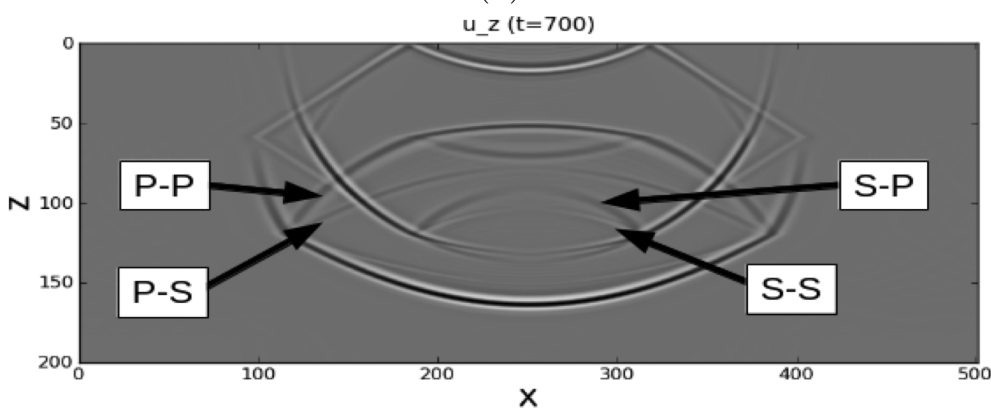

(c)

Figure 2. Snapshots of a simulation of a model consisting of a water layer with a depth of 60 above a layer over a halfspace where the halfspace interface is at a depth of 120 . Mode conversions such as $P-P, P-S, S-P$ and $S-S$ are visible in the solid layers below z=60 whereas only $P$-waves exist in the water layer above $\mathrm{z}=60$.

Figure 5 shows the error versus iteration for this example and indicates that after 100 iterations, almost all of the energy is modelled (ie. the error approaches zero). The initial residuals and the residuals after 100 iterations are shown at the same scale in Figure 6. After 100 iterations, there is no visible energy left in the residuals again indicating that the simulated data through the inversion result nearly perfectly matched the simulated data through the diffractors model. 


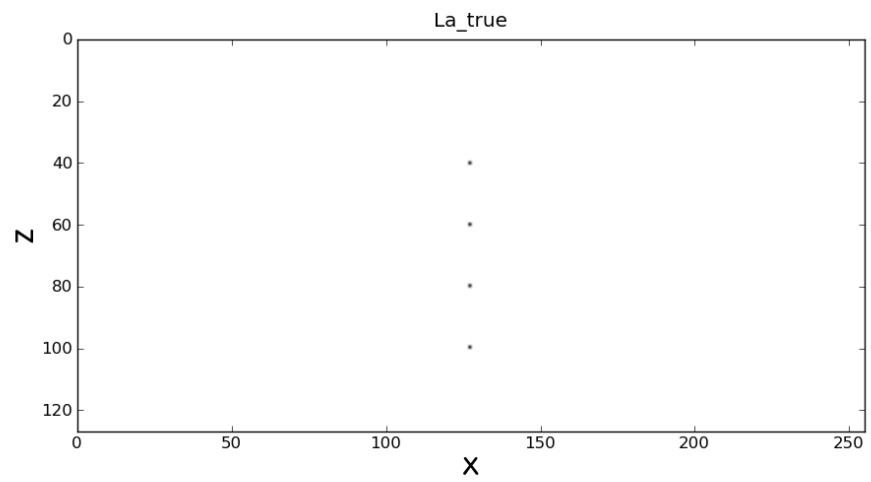

(a)

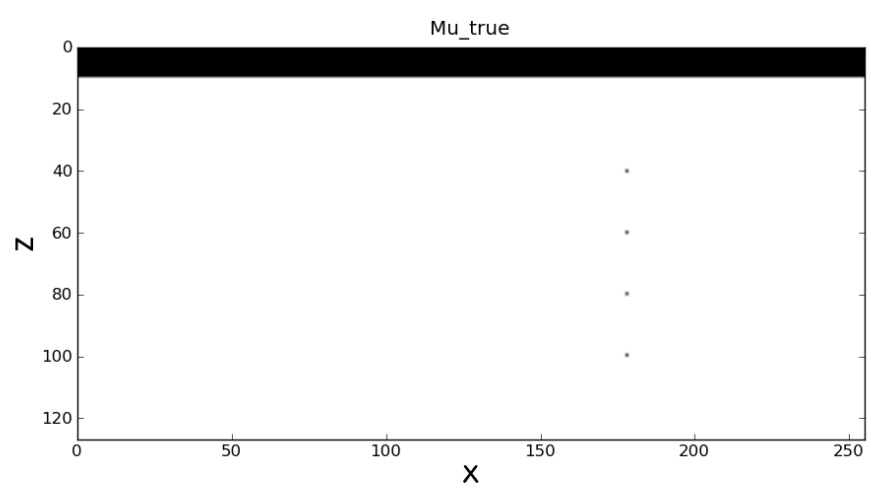

(b)

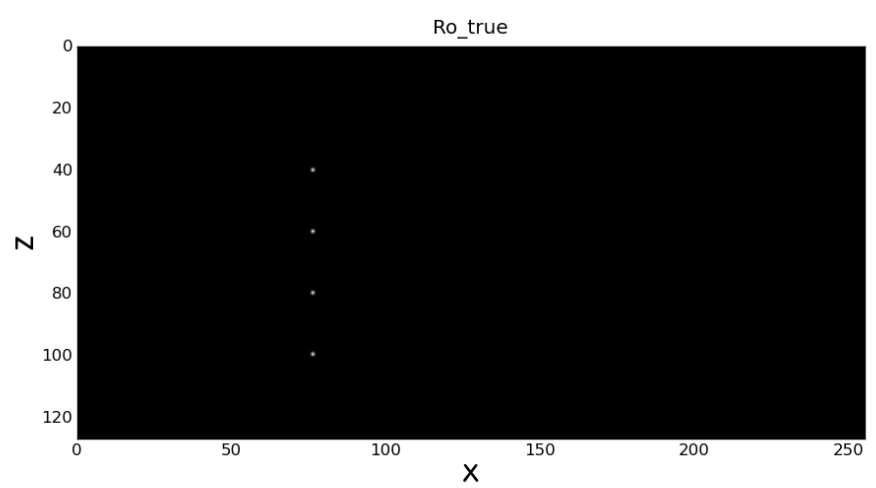

(c)

Figure 3. 2D diffractors model: first Lamé parameter $\lambda$ (a), second Lamé parameter $\mu$ (b), and density $\rho(\mathrm{c})$. 


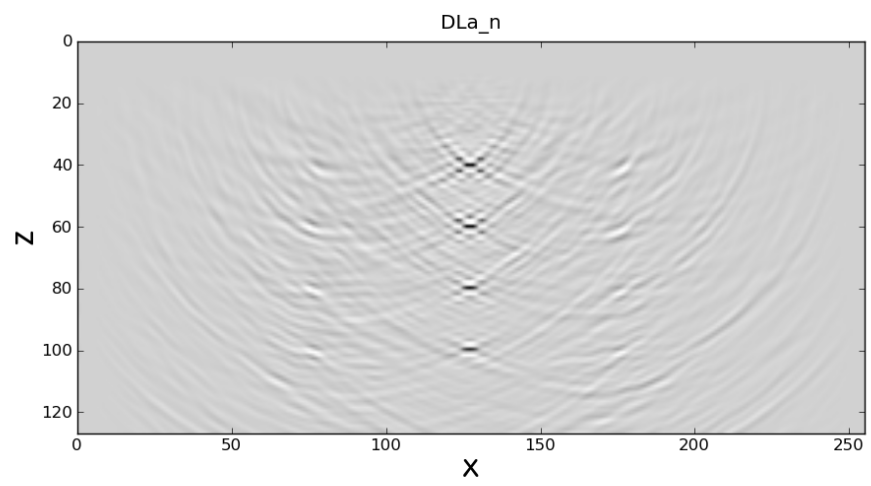

(a)

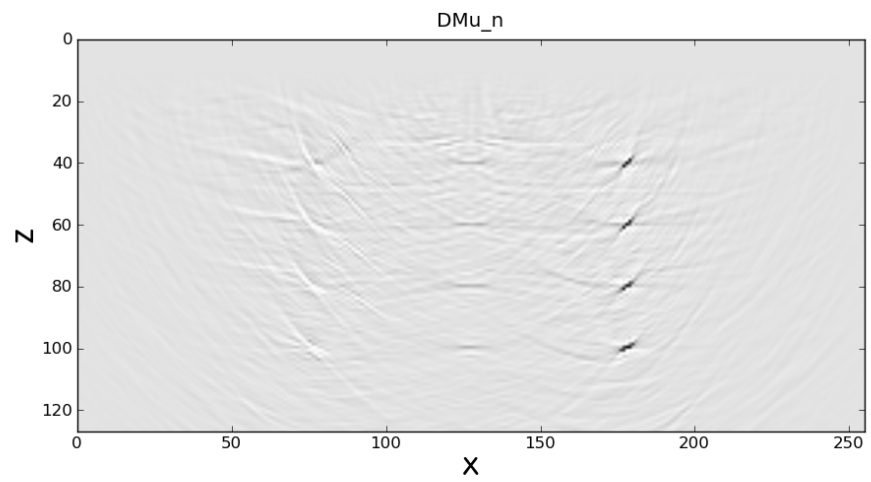

(b)

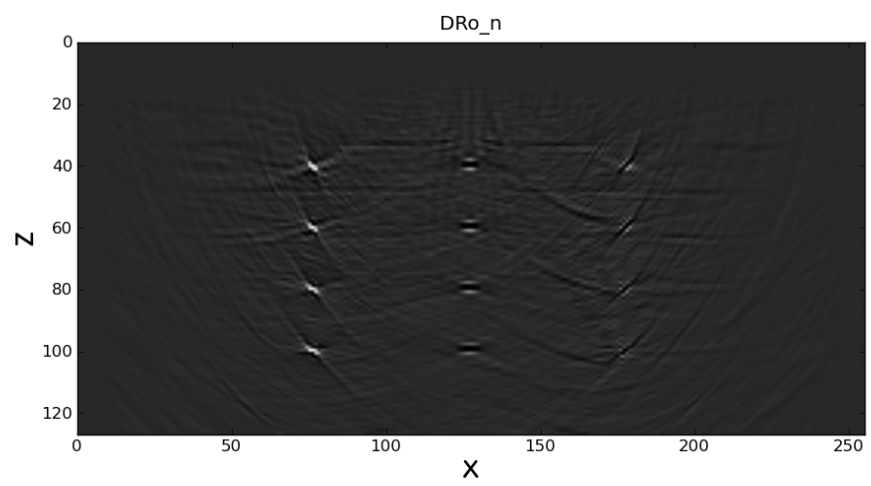

(c)

Figure 4. The diffractors model single shot elastic inversion result of elastic marine data after 100 iterations: first Lamé parameter result $\lambda_{100}$ (a), second Lamé parameter result $\mu_{100}$ (b), and density result $\rho_{100}(\mathrm{c})$. 


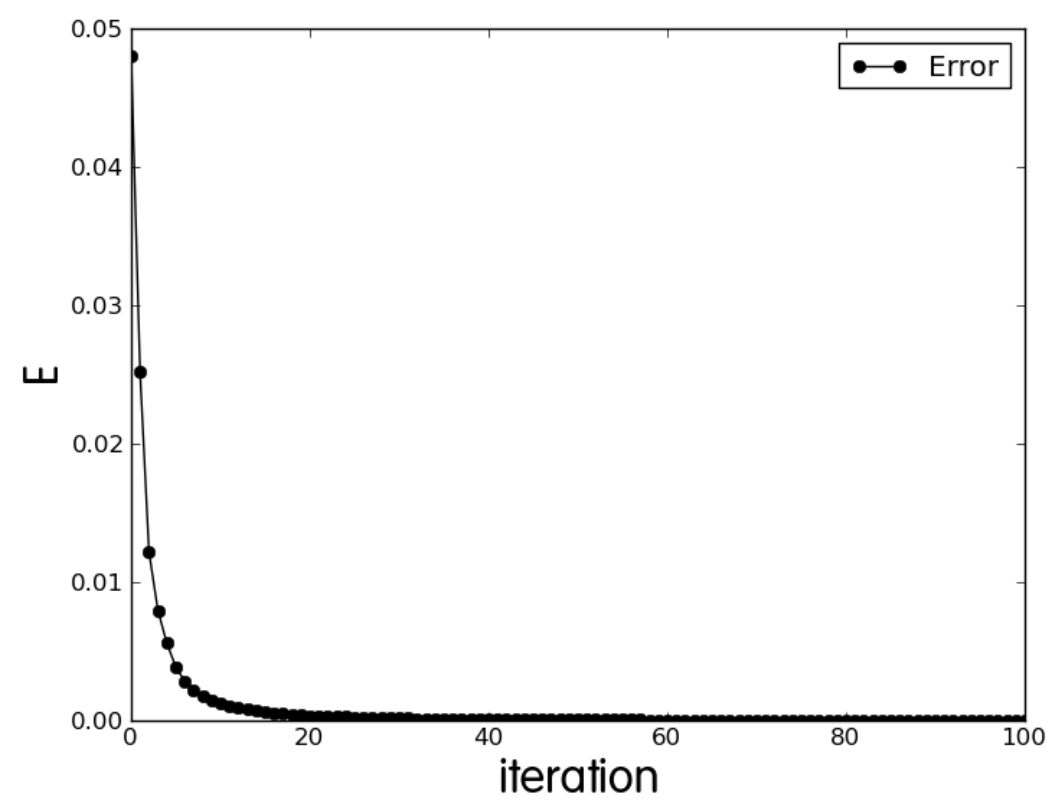

Figure 5. The error versus iteration for the elastic inversion of elastic marine data for the diffractors model.

\subsection{Acoustic inversion of diffractors - single shot}

In this section, we investigate application of an acoustic inversion of the elastic wavefield data, which is the same as previous section. We found that when we aplied an acoustic inversion to the fully elastic data, that most of the effort was spent trying to futilely correct the seafloor bottom reflection. As such, we stripped the seafloor bottom reflection from the simulated data prior to doing the acoustic inversion. This was achieved by doing an elastic and acoustic simulation in a model with the water layer only, and subtracting this water bottom reflection data from the simulated elastic dataset, and from the acoustic simulations carried out during the inversion. In this way, the acoustic inversion only tries to match the data due to diffractions and reflections from within solid layers below the sea-floor, thereby providing a good chance for an acoustic inversion to succeed when inverting for the subsurface below the water bottom. In contrast, the water bottom reflection was not stripped from the elastic inversion example as it was automatically modelled correctly because the starting model had the correct water depth and elastic properties.

The diffractors and results are shown in Figures 7 and 8. The first iteration results show the four diffractors in $\lambda$ in the correct positions, albeit a lower frequency image than obtained in the elastic inversion. The density image also shows four perturbations located at the four 


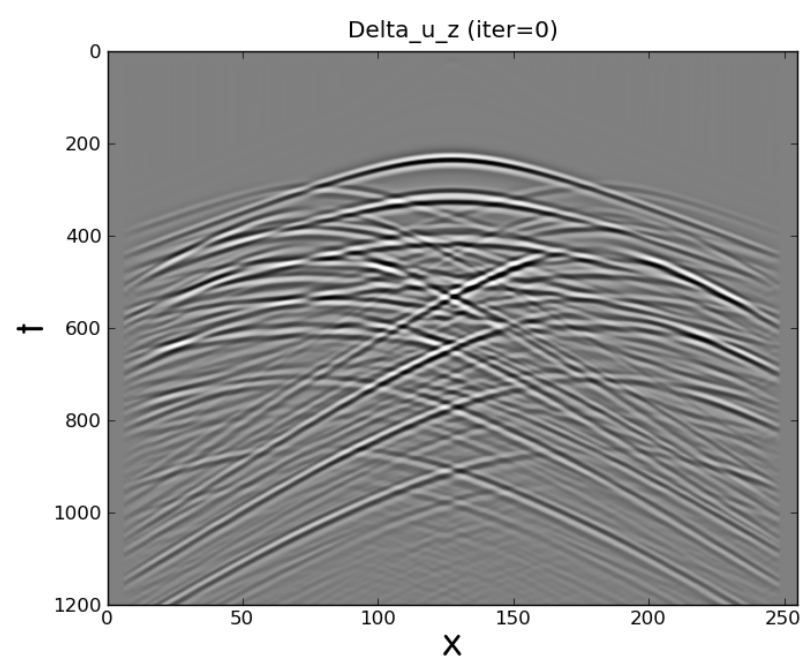

(a)

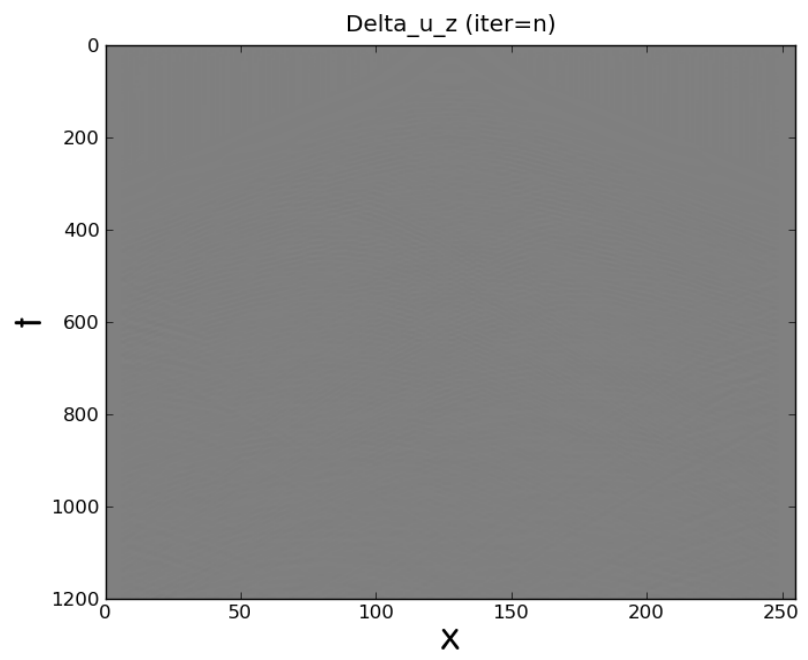

(b)

Figure 6. The diffractors model initial residuals (a) and the residuals after 100 iterations for the elastic inversion of elastic marine data.

positions of the $\lambda$ diffractors. Hence, after one iteration, the acoustic inversion was only able to correctly resolve the diffractors in $\lambda$, but was unable to resolve the density diffractors. Furthermore, after 100 iterations, the initial result for $\lambda$ was degraded with large artefacts appearing throughout the model. This is due to the inversion trying to match the mode converted energy which is not possible using acoustic simulations. As such, the inversion placed artificial diffractors throughout the model such that the overall error, shown in Figure 9, was reduced. One can see from the plot of error versus iteration, that the error only decreased by an additional $25 \%$ from the 5 -th to the 100 -th iteration (ie. from 0.017 to 0.013 ). 
In contrast, the residual in the elastic inversion was already lower than the acoustic case after only 2 iterations, and the residual continued to drop to close to 0 after the 100 iterations.

If one considers the residual for the acoustic case, it is clear why the error could not be reduced much after the first few iterations. One can see from the plot of residuals shown in Figure 10 that the residuals correspond to mode conversions from the $\mu$ diffractors. Such mode conversions are centered at $x_{\mu} \approx 180$ and have a lower velocity (ie. rate of moveout per unit time at large offset $\rightarrow S$-velocity) than the diffractions centered at $x_{\lambda}$. However, the acoustic inversion does not model such mode conversions, and therefore, it cannot resolve this different rate of moveout with time as a diffractor. Rather, it attempts to create $\lambda$ and $\rho$ perturbations that emulate the effect of the mode converted diffractions.

Note that a test was also conducted of a supershot gather consisting of 18 different shot locations. The result was similar to the single shot result, and for this reason, has not been included here. And worse, the more iterations that are made, the worse the result becomes due to the inversion adding artificial perturbations in the model to try to emulate the effect of the $\mu$ diffractors.

Note that the absence of any perturbations in the inversion images above 10 units deep is due to energy in the gradient being muted out in the water layer which is considered as a known region of the model. In the case of the acoustic inversion of elastic data, to give the best possible chance to the acoustic inversion, we stripped the water bottom reflection, and hence, we can consider that in our case, the model is known perfectly down to the start of the solid layer below the water bottom.

\section{ELASTIC INVERSION OF ELASTIC MARINE DATA (FAULT MODEL)}

A model was defined consisting of a simple Gaussian anticline with two thrust faults as shown in Figures 11a through 12a. The layer properties for $\mu$ were independent to those of $\lambda$. For example, the low $\lambda$ layers were layers 3 (dark blue) and 7 (blue between two yellow layers), cf. the low $\mu$ layers were layers 4 (bright blue) and 8 (aqua-green below yellow). The layer properties are shown in Table 1. Note that from the layer properties, one can see that the maximum values of the moduli are $1.4 \times$ the amplitude of the lowest values of unity. This corresponds to $V_{p}$ and $V_{s}$ ranging up by $20 \%$ from the shallowest to deepest layer. This is a somewhat smaller range than normal. However, this small range was necessary due to limited computer resources (a home workstation). A supershot consisting of 28 pressure sources equally spaced along the surface of the model was simulated using the staggered finite-difference scheme. The start times of each shot were randomized to eliminate artificial correlations in the model 


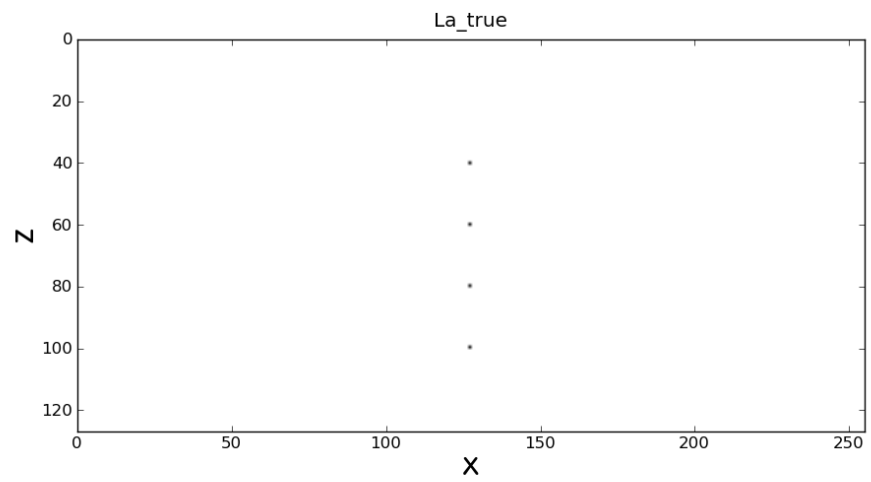

(a)

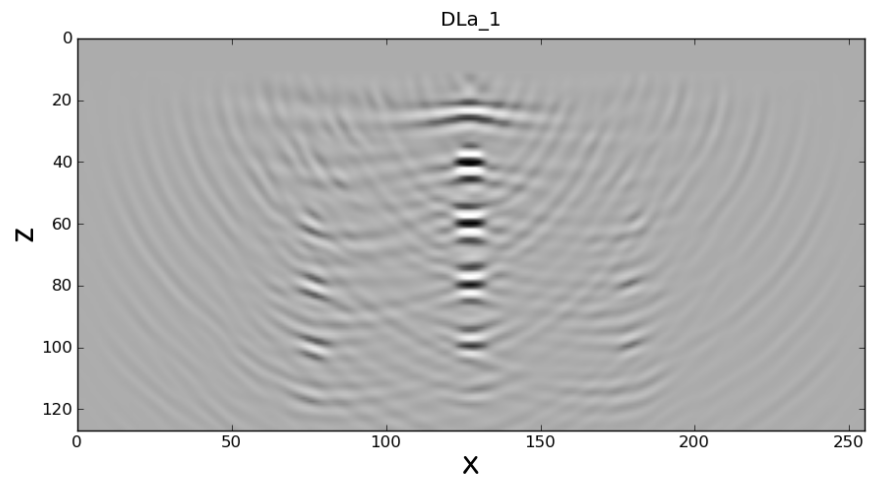

(b)

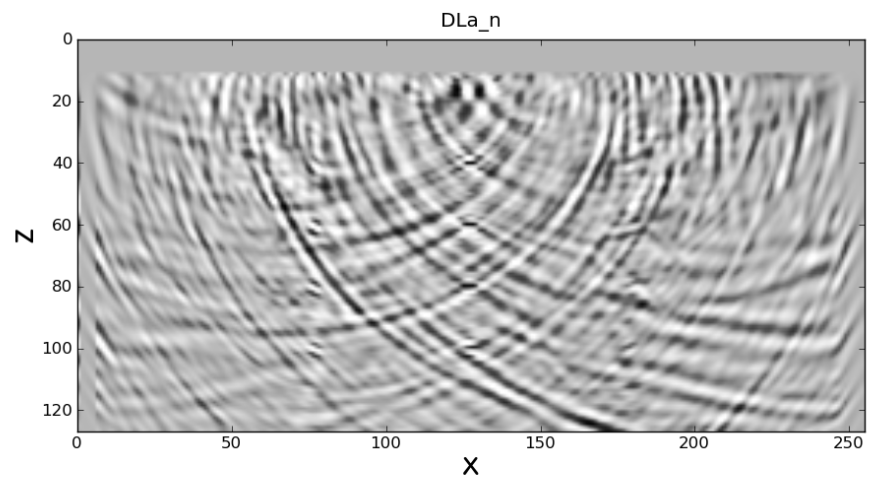

(c)

Figure 7. A 2D diffractors model of the first Lamé modulus $\lambda$ of the subsurface (a), and the acoustic inversion result of elastic marine data after 1 iteration (b), and after 100 iterations (c). The water layer has a value of $\mu_{\text {water }}=0$ and hence, can be seen as a black band at the top of the model in (b). The diffractors are point perturbations in the model at four different depths $(z=40,60,80$, and 100). The locations of the diffractors are as follows: density diffractors at $x=76.5, \lambda$ diffractors at $x=128$, and $\mu$ diffractors at $x=178.5$. 


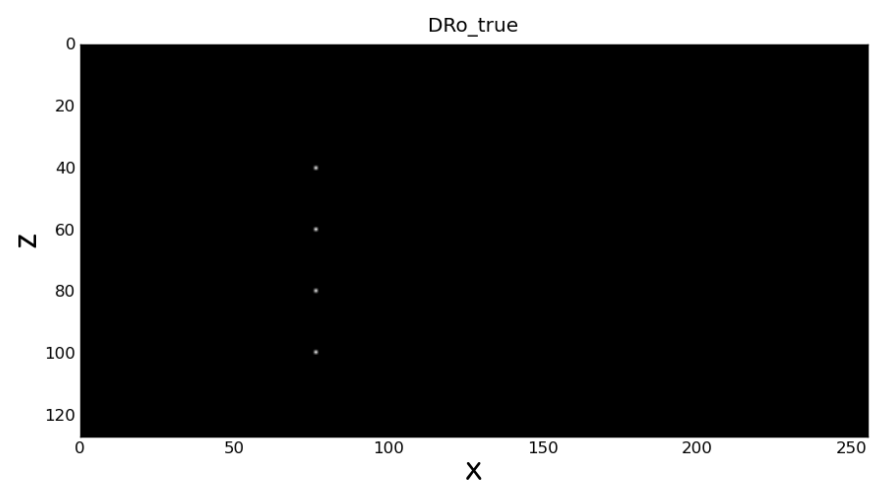

(a)

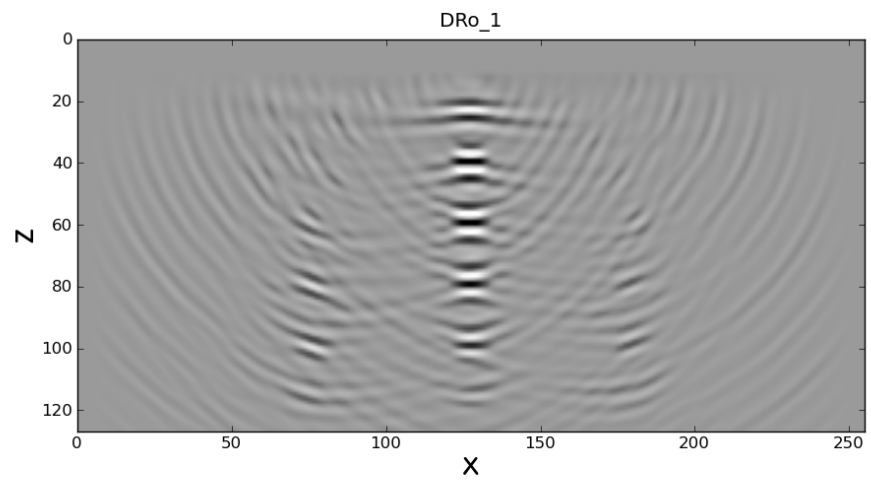

(b)

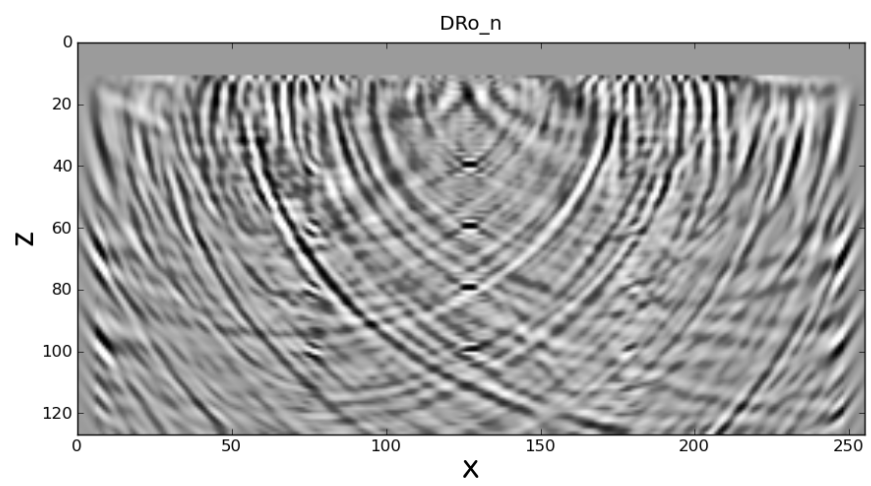

(c)

Figure 8. A 2D diffractors model of the density $\rho$ of the subsurface (a), and the acoustic inversion result of elastic marine data after 1 iteration (b), and after 100 iterations (c). 


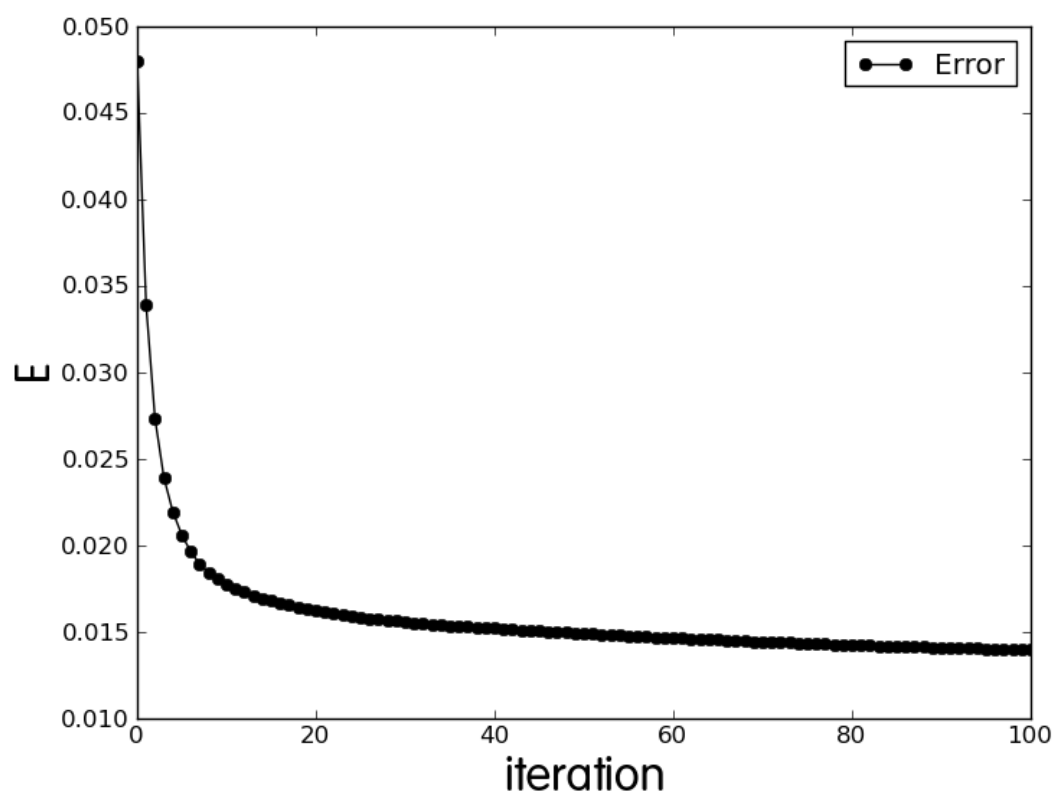

Figure 9. The error denoted E versus iteration for the acoustic inversion of elastic marine data for the diffractors model.

due to pre-summing of the shots into a supershot. This randomization of source times was re-done every two iterations of the conjugate gradient algorithm to minimize the correlations between the shots (see also, Schiemenz \& Igel (2013)). An example of a single shot profile for data simulated in the model is shown in Figure 13. One can see seafloor multiples and strong $P-P$ reflections at zero offset and extending to large offsets. In addition, one can see the slow direct water $P$-wave, and following behind the direct $P$-wave, one can observe weak reflections with a similar velocity to the water velocity, and with zero amplitude at zero offset. These reflections are $P-S-S-P$ modes and are what allow the shear modulus to be resolved. Figure 14 shows a 28 shot supershot gather for the fault model which represents the dataset used to perform the inversion.

Our goal here is to compare elastic inversion of elastic data with acoustic inversion of elastic data under ideal circumstances for both. As such, periodic boundary conditions were used in the $x$-direction and 1500 time steps were calculated of the finite-difference simulation of elastic waves on a $502 \times 232$ sized finite-dfference mesh. This is enough to record seismic waves to large offsets with ratios of offset to depth in the range 1:1 to $3: 1$. Also, as the aim is to identify artefacts due to using an acoustic inversion of elastic data, and has nothing to do with quantification of acoustic or elastic inversion results, no colour scales are provided in the following sections as these would distract the reader from this aim. Furthermore, due to the 


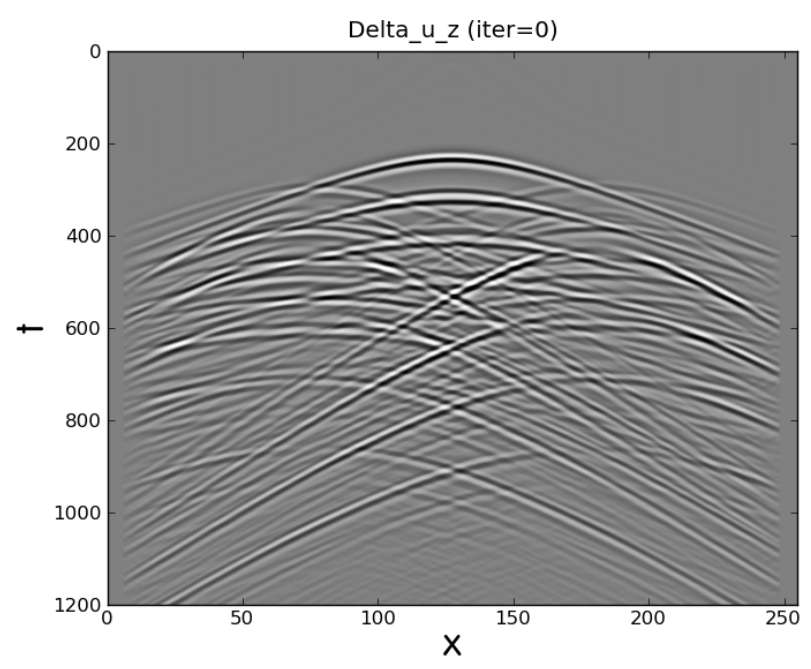

(a)

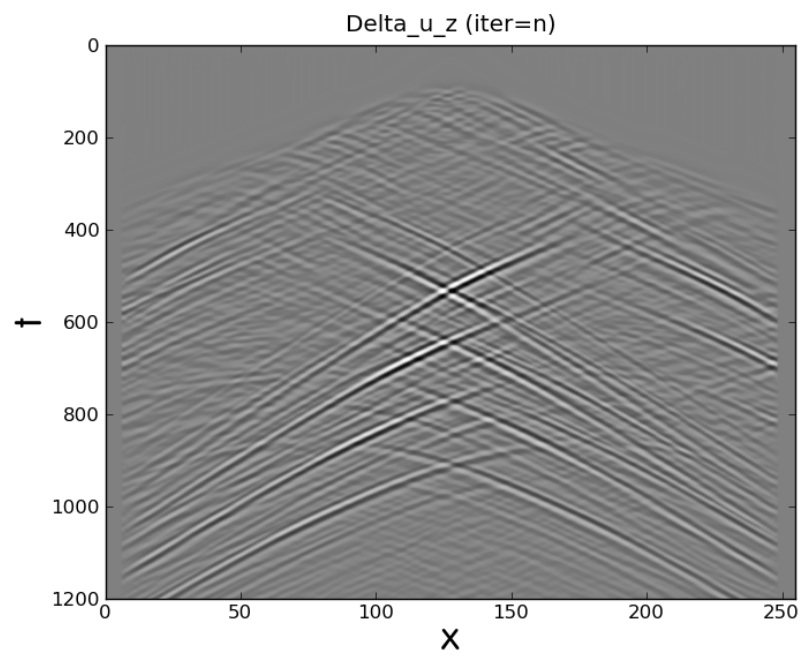

(b)

Figure 10. The diffractors model initial residuals (a) and the residuals after 100 iterations for the acoustic inversion of elastic marine data.

large contrast in elastic parameters in the water layer versus the rest of the model, a standard rainbow colour scheme would mainly visualizes the water layer, with almost no dynamic range left to visualize the elastic properties in the solid layers, or the soft waterbottom layer. In the following, dark blue is used to visualize the water layer and bright blue is used to visualize the soft sediments layer, whereas a standard colour rainbow is used to visualize the elastic parameters in the solid layers. As is typically done for idealized test case inversions, we set the initial models to be smoothed versions of the exact models as shown on Figures 11b and 12b.

Figures 11c through 15 show the results of an inversion after 200 iterations of a conjugate 
gradient algorithm (Note: the residuals after 200 iterations were small and hence, the inversion was stopped at 200 iterations). These results show that $\lambda$ and $\mu$ are well resolved from one another, but the density $\rho$, which should be constant, has a ghost image of the Lamé parameter (see Figure 15). This is due to imperfect resolvability of $\rho$ from $\lambda$ and $\mu$ as was also seen in the inversion of the diffractors.

Figure 16 shows the error as a function of iterations and demonstrates that most of the energy has been matched after the 200 iterations. Note that the zig-zag pattern of error up and down is due to the source randomization procedure every second iteration. While this randomization procedure seems to slow the convergence relative to a case where source times are left constant, tests have shown that this randomization leads to clearer images. Namely, the time randomization of shots when summing the shots into supershots yields images without artefacts due to source correlations (i.e. from source cross-talk) which occur if one sums the shots into a supershot with a fixed timing. Hence, rather than slowing down an inversion, the pre-summing of shots into a supershot speeds up the inversion by a factor approaching the number of shots when compared to an inversion of individual shots.

Figure 17 shows the initial residual, the initial supershot gather with the seafloor bottom reflection stripped, compared to the residual after 200 iterations. This plot shows that the final residual is close to zero and hence, the inversion has matched most of the data in the supershot record.

\section{ACOUSTIC INVERSION OF ELASTIC MARINE DATA (FAULT MODEL)}

In this section, we wish to apply an acoustic inversion to the data calculated in the elastic model. That is, using the same elastic data as was calculated in the previous section, we perform an acoustic inversion. In this case, to ensure the physics of the inversion is comparable to the elastic case, we have initialized the acoustic model to the $P$-wave modulus denoted $M=\lambda+2 \mu$, and performed finite-difference calculations using this $P$-wave modulus in place of $\lambda$, and with a shear modulus $\mu=0$. Namely, we define $\lambda_{a}$ and $\mu_{a}$ as the acoustic Lamé parameter. These are related to the elastic Lamé parameters $\lambda=\lambda_{e}$ and $\mu=\mu_{e}$ as follows

$$
\begin{aligned}
& \lambda_{a}=M=\lambda_{e}+2 \mu_{e} \\
& \mu_{a}=0 .
\end{aligned}
$$

Hence, $P$-waves will propagate with the same speeds in the acoustic and elastic models, but no shear waves will propagate in the acoustic model.

The elastic model used to calculate the data is shown in Figures 11a to 15. However, 


\begin{tabular}{cccccc}
\hline Layer & $\lambda_{e}$ & $\mu_{e}$ & $\rho_{e}=\rho_{a}$ & $M=\lambda_{a}=\lambda_{e}+2 \mu_{e}$ & $\mu_{a}$ \\
\hline water & 1 & 0 & 1 & 1 & 0 \\
1 & 1 & 1 & 1 & 3 & 0 \\
2 & 1.06 & 1.07 & 1 & 3.2 & 0 \\
3 & 0.95 & 1 & 1 & 2.95 & 0 \\
4 & 1.04 & 0.95 & 1 & 2.94 & 0 \\
5 & 1.08 & 1.04 & 1 & 3.16 & 0 \\
6 & 1.22 & 1.08 & 1 & 3.38 & 0 \\
7 & 1.05 & 1.22 & 1 & 3.49 & 0 \\
8 & 1.25 & 1.05 & 1 & 3.35 & 0 \\
9 & 1.4 & 1.4 & 1 & 4.2 & 0 \\
10 & 1.32 & 1.32 & 1 & 3.96 & 0 \\
\hline
\end{tabular}

Table 1. Lamé parameters and density for the elastic 2D fault model and its equivalent acoustic 2D fault model. Values of the elastic model Lamé parameters $\lambda=\lambda_{e}$ and $\mu=\mu_{e}$ (first 2 columns), and the acoustic model Lamé parameters $\lambda_{a}, \mu_{a}$ (last 2 columns). The density $\rho$ (middle column) is the same for both the elastic and acoustic models. The column denoted $M=\lambda+2 \mu$ represents the modulus for pressure waves, which is what can potentially be resolved by an acoustic inversion. Notice that the values of $\lambda$ and $\mu$ differ so these models are independent of one another. For example, layers 3 and 7 have a low value of $\lambda$ sandwiched between higher $\lambda$ layers, whereas layers 4 and 8 have a low value of $\mu$ sandwiched between higher $\mu$ layers. Note that the $P$-wave modulus denoted $M=\lambda+2 \mu$ has a different character than $\lambda$ and $\mu$, with layers 3 and 4 having somewhat lower $M$ than the surrounding layers.

what is potentially resolvable by the acoustic inversion, is the $P$-wave modulus $M$. As such, the true acoustic model that is potentially resolvable by an acoustic inversion is shown in Figure 18a through 19. The scales are different for $\lambda_{a}=M$ compared to $\lambda_{e}=\lambda$ as per Table 1 (ie. $\lambda_{a}=M \approx 3 \lambda_{e}$ ). Again, the initial model is set to be a smoothed version of what can potentially be resolved, which in this case, is the exact model for $M=\lambda_{a}$ as shown on Figure 18b.

Figures $18 \mathrm{c}$ and 19 show the results of an acoustic inversion after 200 iterations of a conjugate gradient algorithm applied to the water-bottom reflection stripped data. These results show that $\lambda_{a}$ is fairly well resolved although it contains cross hatching artefacts and is less clean than the elastic inversion result. Again, the density $\rho$, which should be constant, has a ghost image of the Lamé modulus $\lambda_{a}$ due to imperfect resolvability of $\rho$ from $\lambda$ as was also seen in the inversion of the diffractors, and the elastic inversions. 


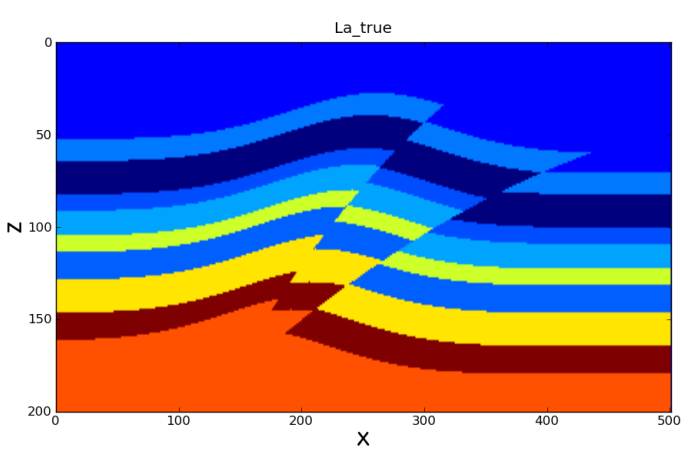

(a)

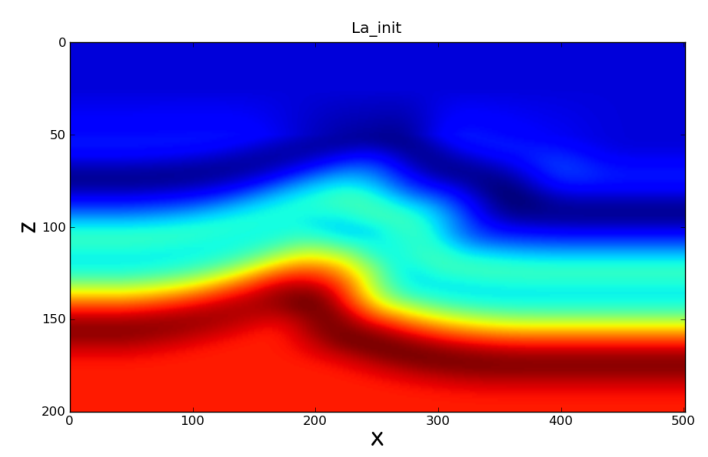

(b)

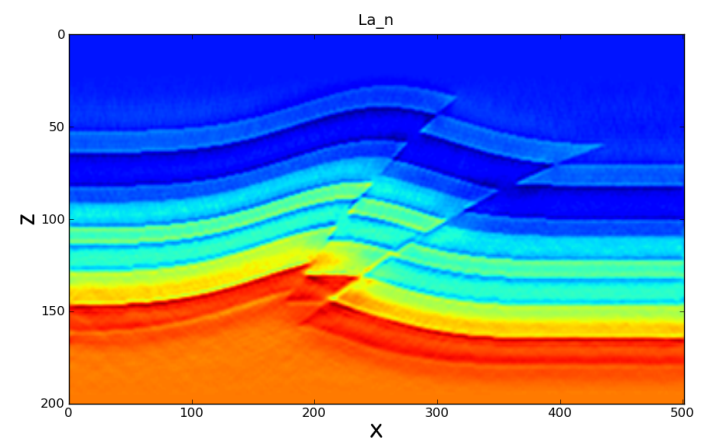

(c)

Figure 11. A 2D fault model of the first Lamé modulus $\lambda$ of the subsurface (a), initial model (b), and the elastic inversion result of elastic marine data after 200 iterations (c).

Figure 20 shows the error as a function of iterations and demonstrates that only a fraction of the energy has been matched after the 200 iterations by the acoustic inversion. Again, the zig-zag pattern of error up and down is due to the source randomization procedure every second iteration. Figure 21 shows the initial residual, the initial supershot gather with the seafloor bottom reflection stripped, compared to the residual after 200 iterations. This plot shows that the residual is far from zero and hence, the inversion has not matched the data 


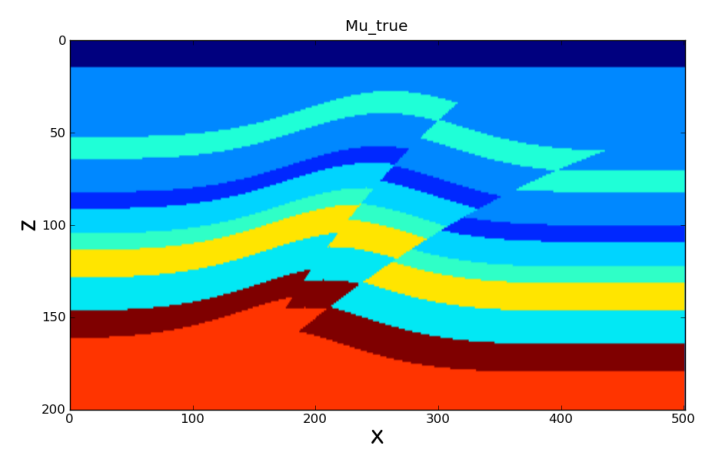

(a)

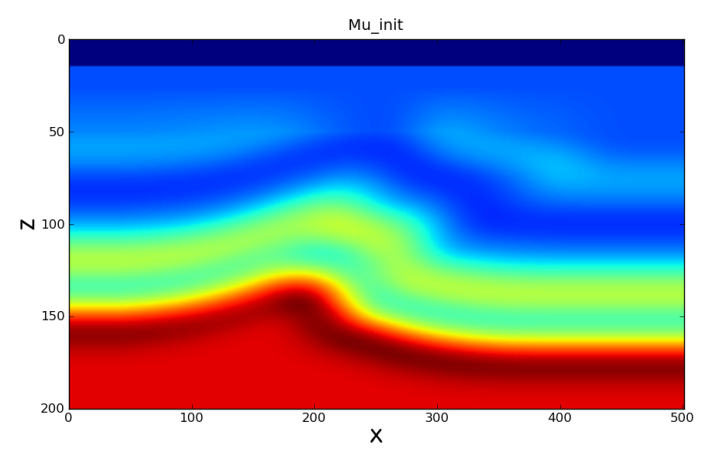

(b)

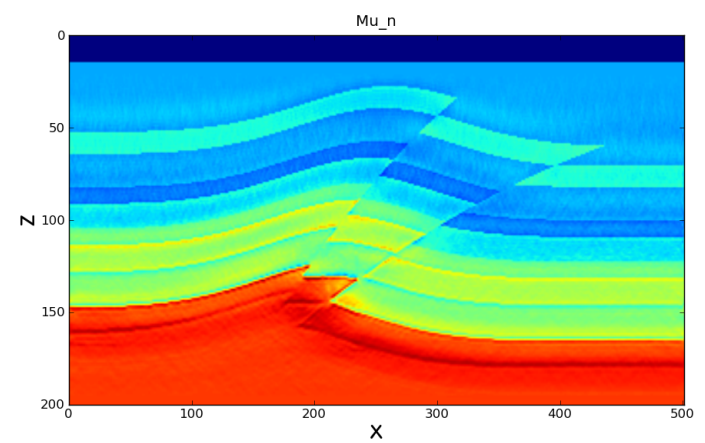

(c)

Figure 12. A 2D fault model of the second Lamé modulus $\mu$ of the subsurface (a), initial model (b), and the elastic inversion result of elastic marine data after 200 iterations (c). The value of $\mu$ in the upper water layer is $\mu_{\text {water }}=0$ (see Table 1 ).

in the supershot record well. The high residual is due to mode converted energy, as was the case for the diffractors inversion result. Again, the $S$-wave mode conversions cannot be well matched. As such, artefacts are visible on the inversion result for $\lambda_{a}$. 


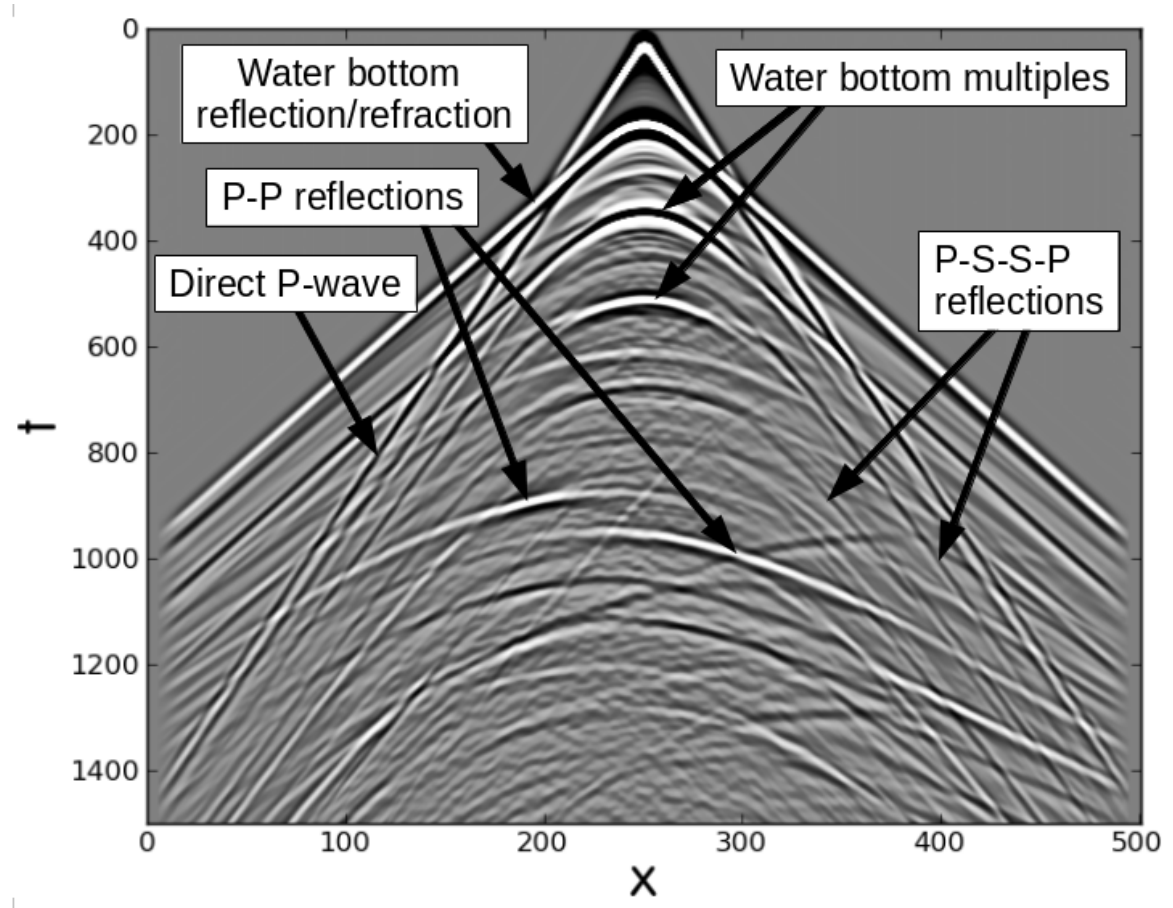

Figure 13. A single shot gather due to a source located in the centre of the fault model shown in Figures 11a and 12a simulated using absorbing boundaries. The supershot gather consisted of 28 such shots.

\section{ACOUSTIC INVERSION OF ACOUSTIC MARINE DATA (FAULT MODEL)}

In this section, we apply an acoustic inversion to data calculated in an acoustic model, cf. the last section was an acoustic inversion of elastic data and hence, was intended as an idealized acoustic inversion applied to real data, which by definition, is elastic. The reason for doing this last artificial case is for completeness, and to resolve whether the acoustic inversion has some intrinsic limitation. Specifically, are the artefacts due to doing an acoustic inversion such as seen in previous sections when the data is calculated with the same equation (acoustic wave-equation) as is used to do the inversion. Or are the artefacts due in some way related to the use of acoustic waves?

Figure 22 through 25 show results of an acoustic inversion of acoustic data after 200 iterations. Namely, acoustic waves were simulated in the $P$-wave modulus version of the fault model (see Table 1), and this acoustic data was then inverted using acoustic inversion (ie. acoustic simulations were used to calculate the gradient in $M=\lambda_{a}$ and $\rho$ ). Figure 22 shows that the image for $\lambda_{a}$ is now sharp and is of similar quality to the $\lambda_{e}$ result of the elastic inversion of elastic data (see Figure 11c). Hence, the artefacts and lower quality images for 


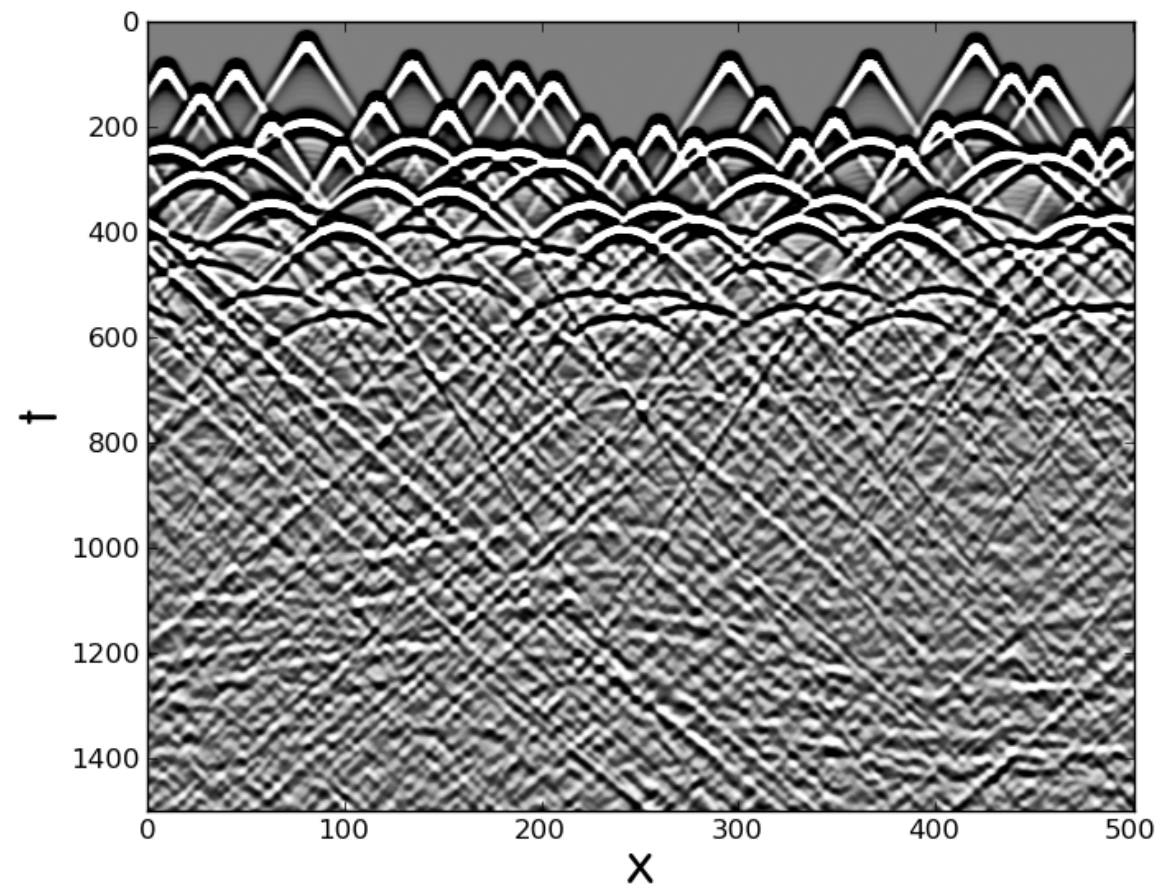

Figure 14. The 28 shot supershot gather for the fault model shown in Figures 11a and 12a. The supershot was calculated as a single simulation with 28 sources located at a regular increment along the top of the model and triggered at random start times. The boundary conditions were circular in the $x$-direction to efficiently simulate the equivalent case of summing 28 large offset gathers without any edge effect.

the acoustic inversions of elastic data are entirely due to using the wrong wave-equation to invert the data. When we say wrong, we mean the approximate acoustic wave-equation that is unable to simulate all elastic wave phenomena in the data such as $P-S$ mode conversions.

Figure 24 shows the error versus iteration for the acoustic inversion of acoustic data, and Figure 25 shows the initial and final data residuals. Whereas in the acoustic inversion of elastic data, a significant amount of energy could not be matched, the error of the acoustic inversion of acoustic data converged to almost zero after the 200 iterations (Figure 24), and the data residuals became small (of similar amplitude as for the case of elastic inversion of elastic data - Figure 17).

Based on these results, the lower quality inversion results and artefacts seen in previous sections on acoustic inversion of elastic data are entirely due to the presence of mode converted energy that cannot be modelled by the acoustic wave-equation. It is not in any way due to any intrinsic limitation of acoustic inversion. 


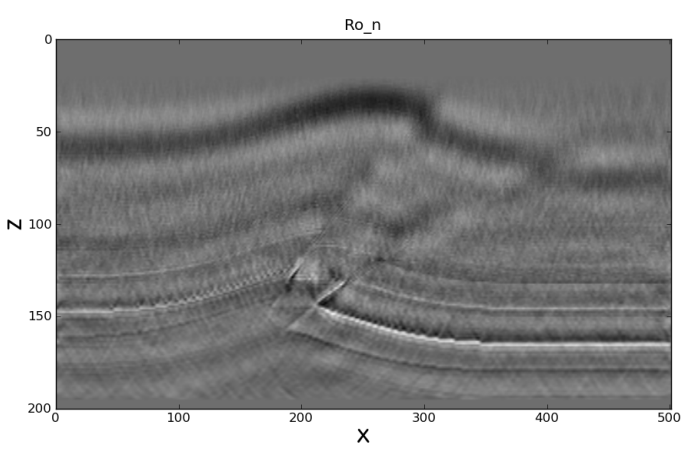

Figure 15. The elastic inversion result for density $\rho$ of the elastic marine data after 200 iterations. The true and starting models for density were both constant with $\rho=1$ and hence, are not shown here.

\section{SOFT SEDIMENTS EXAMPLE}

This section aims to study the effect of a layer of soft sediments on hard waterbottom examples studied so far. The question being posed was "Will the results be the same when a soft sediment layer exists where potentially less converted $S$-wave energy occurs at the sea floor?". The model used was identical to the fault model of previous sections except that a layer under the acoustic (water) layer was added with values of $\lambda=1$ and $\mu=0.25^{2}$, compared to the upper rock layer with $\lambda=\mu=1$. Hence, the $S$-wave velocity of the layer representing soft

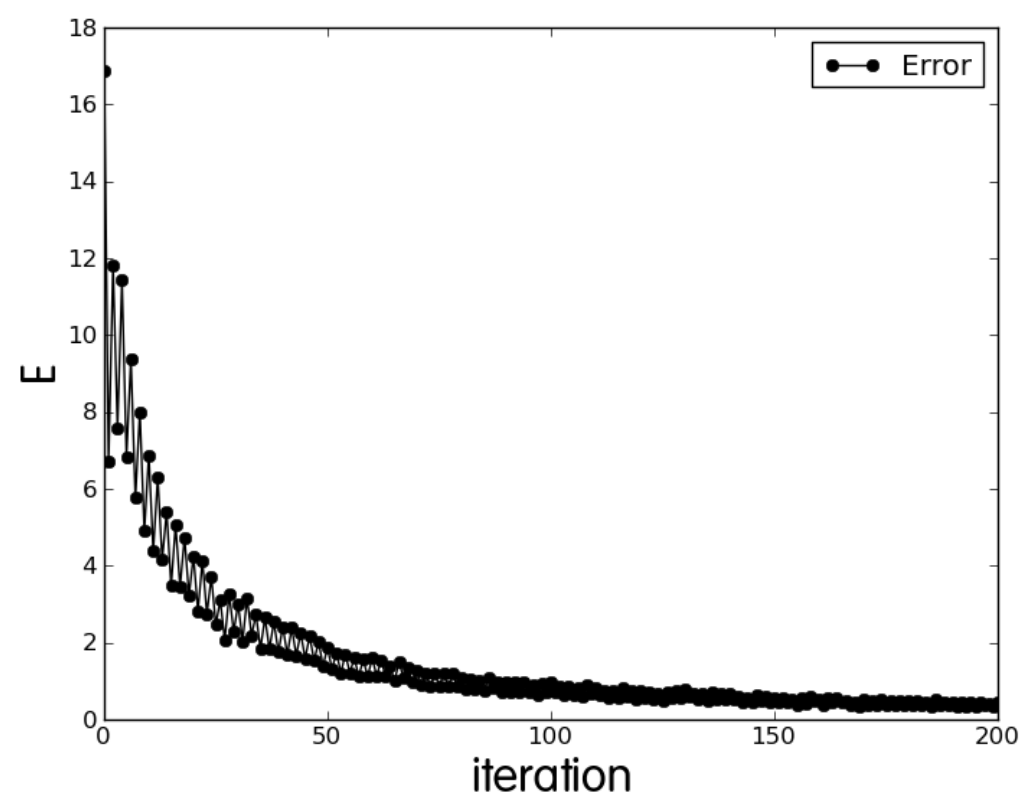

Figure 16. The error versus iteration for the elastic inversion of elastic marine data. 


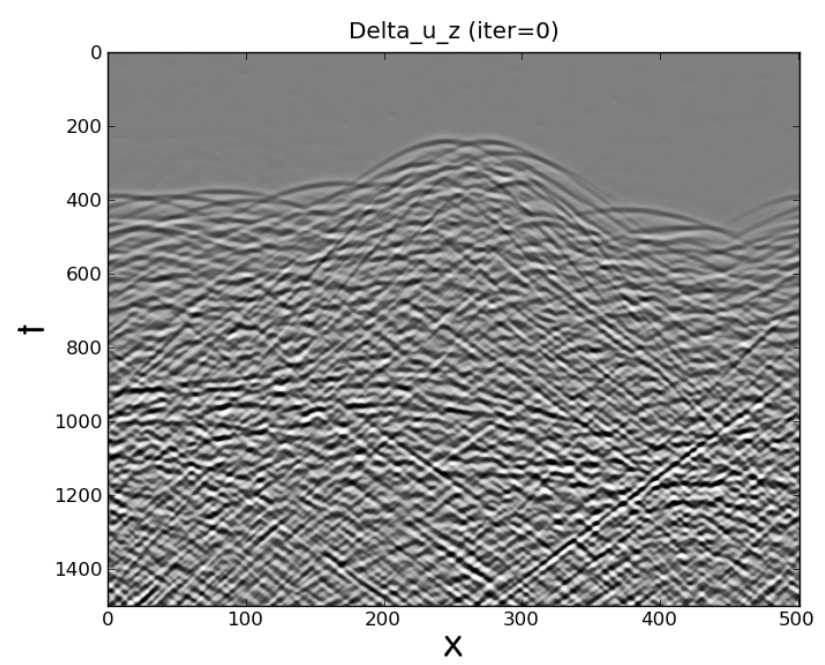

(a)

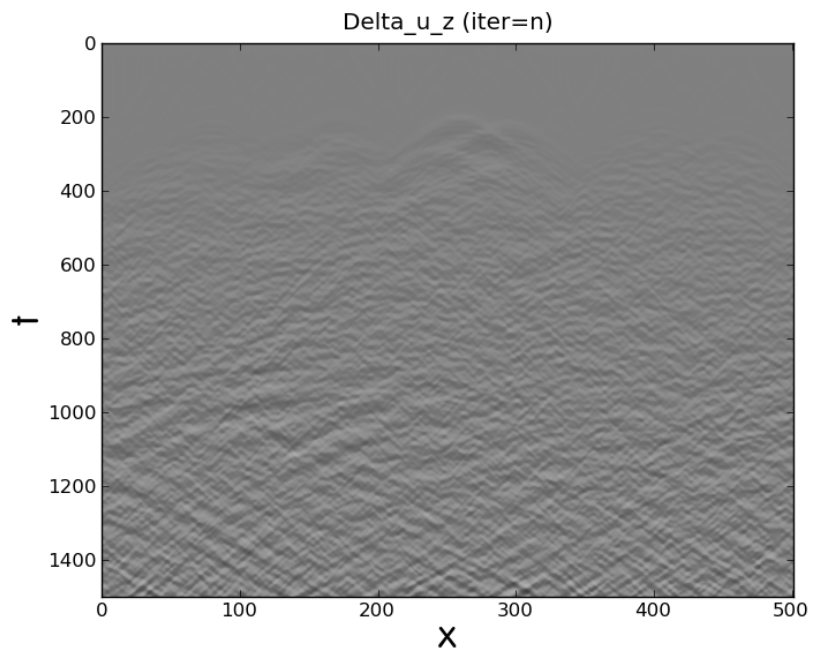

(b)

Figure 17. The initial residuals (a) and the final residuals after 200 iterations (b) for the elastic inversion of elastic marine data.

sediments was $0.25 \times V_{s}($ rock $)$. This layer was 7 gridpoints thick or about one wavelength, and hence, is intended to represent a narrow layer of soft sediments with a low shear wave velocity. While this may be an adequate representation of a soft sediment layer in terms of $P$ and $S$ wave velocities, it does not have attenuation that may be expected of soft sediments, and is therefore potentially not accurately representative.

Figure 26 shows the inversion results after 200 iterations for the three cases run. Namely, elastic inversion of elastic data, acoustic inversion of elastic data, and acoustic inversion of acoustic data. The three sets of results are almost identical to the results of the previous 


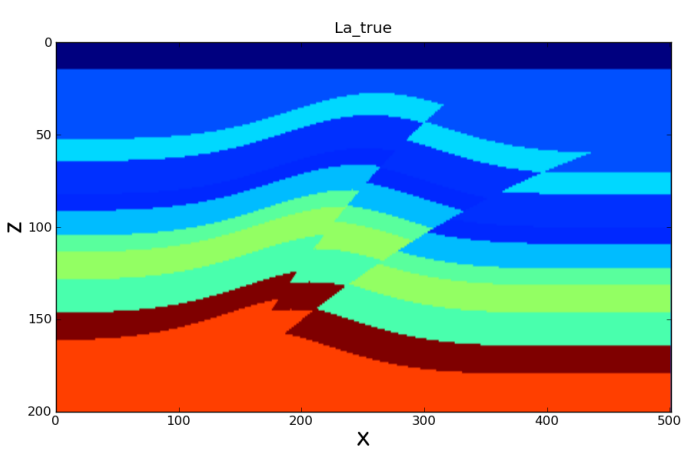

(a)

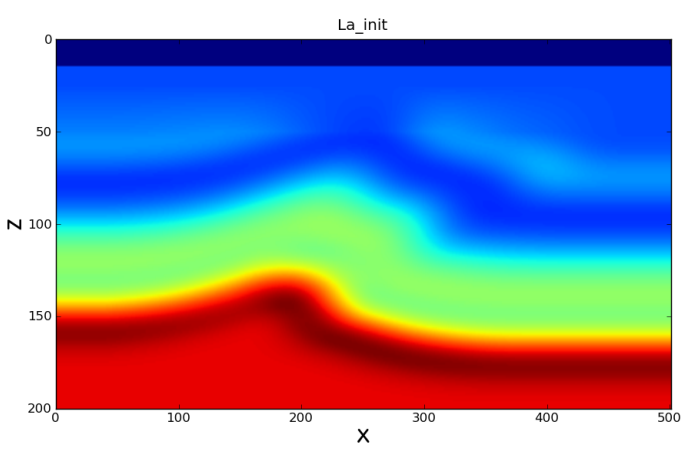

(b)

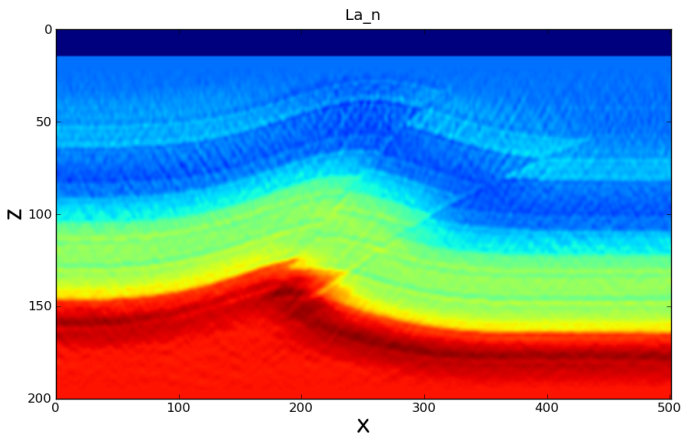

(c)

Figure 18. A 2D fault model of the $P$-wave modulus $=$ first Lamé modulus $M=\lambda_{a}$ of the subsurface (a), initial model (b), and the acoustic inversion result of elastic marine data after 200 iterations (c). This result has a lower clarity than the result of elastic inversion of elastic data shown in Figure 11, with a cross-hatched appearance which are mode-conversion artefacts.

sections for the hard water bottom case. Namely, the elastic inversion of elastic data yields two clean images of the $\lambda$ and $\mu$ whereas the acoustic inversion of elastic data yields one less clean image of $\lambda$ with some artefacts. The final image shown is for acoustic inversion of acoustic data and this image is shown to be clean, demonstrating that the cause of the artefacts in the case of acoustic inversion of elastic data is the mode converted waves. 


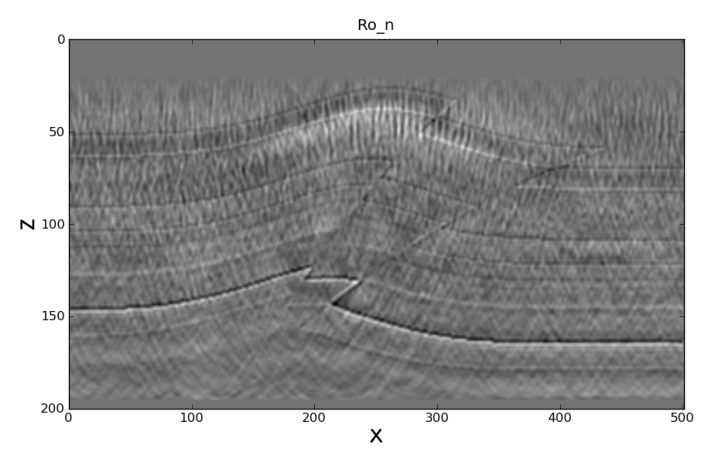

Figure 19. The acoustic inversion result for density $\rho$ of the elastic marine data after 200 iterations. The true and starting models for density were both constant with $\rho=1$ and hence, are not shown here.

\section{DISCUSSION}

The results presented in the previous sections demonstrate that when applying acoustic inversion to elastic data, artefacts are severe for diffraction data, whereas artefacts for layer reflections are minor. We interpret this as follows. Consider a layer result as the sum of diffractor results where diffractors are placed at all points in a layer. Hence, consider a thin reflector result as the sum of the diffractor results where the diffractors are shifted in $x$. In this case, one will obtain a coherent signal at the depth of the thin reflector. However, the

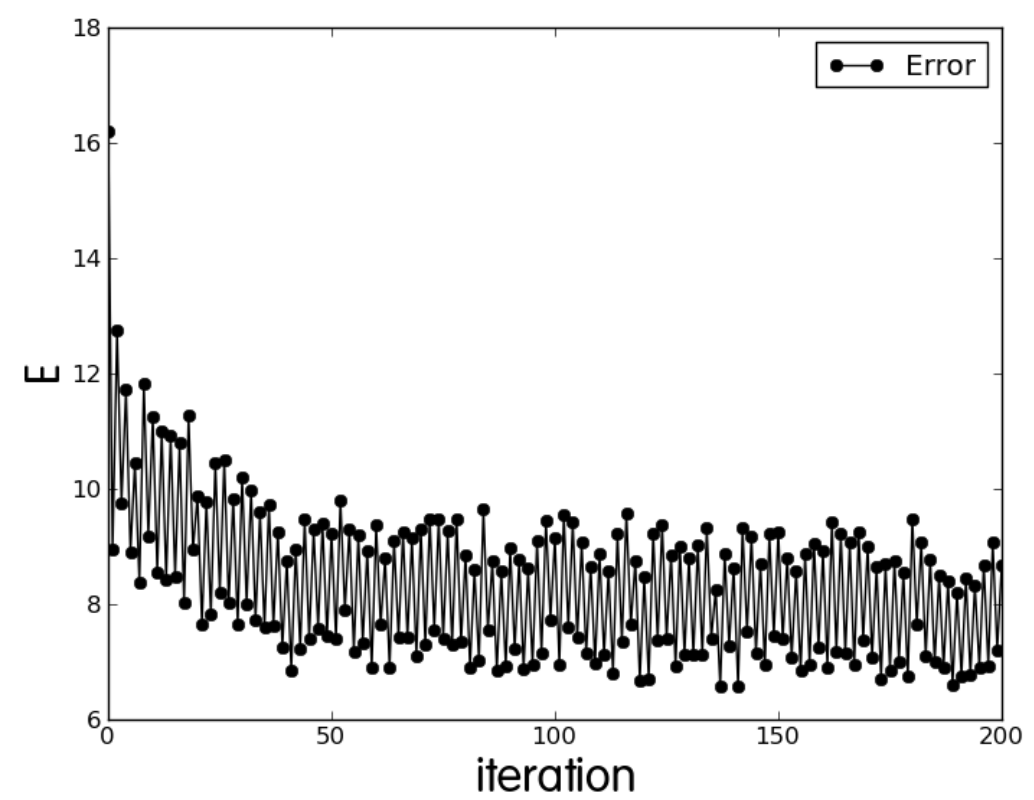

Figure 20. The error versus iteration for the acoustic inversion of elastic marine data. 


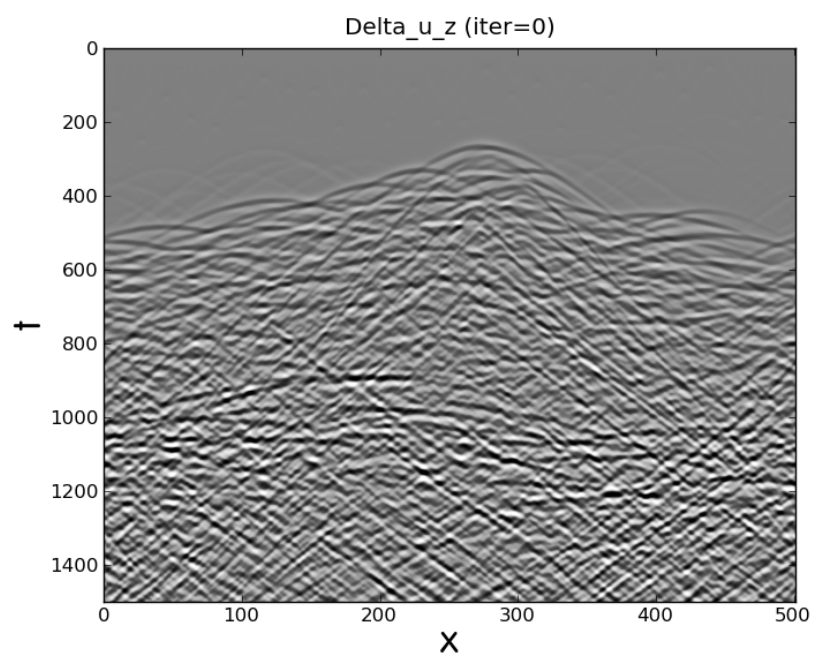

(a)

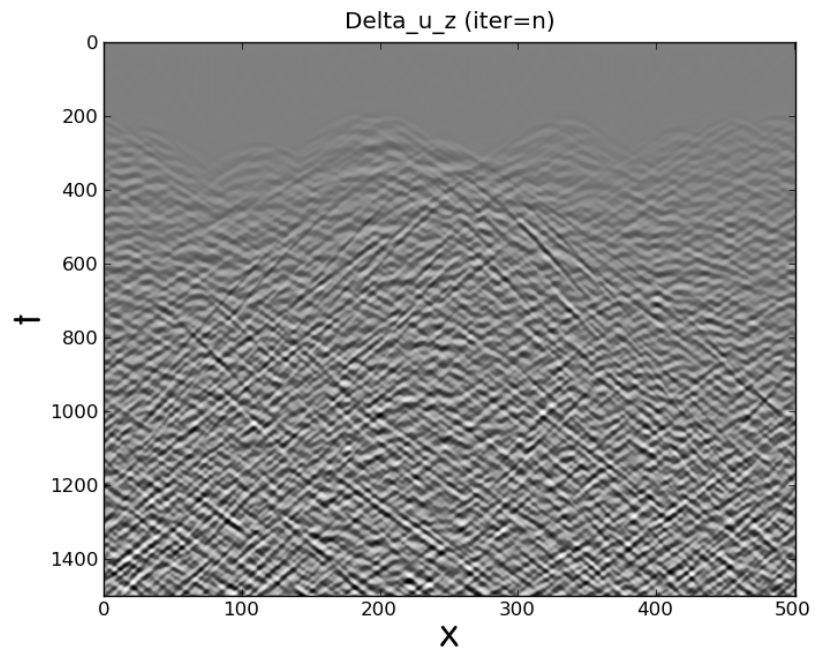

(b)

Figure 21. The initial residuals (a) and the final residuals after 200 iterations (b) for the acoustic inversion of elastic marine data.

artefacts, which are oscillatory events that are similar to migration "smiles", are summed but these largely cancel out due to having a zero mean. A layer result would then be the sum of thin reflector results where the depth of the thin reflector varies from $z_{\text {reflector }}$ to

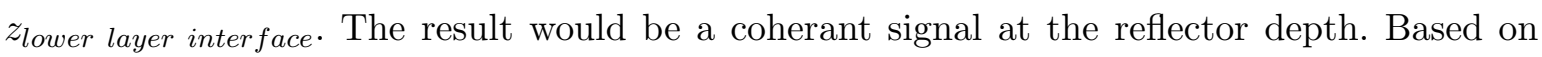
the results of this paper and the above interpretation, one can expect that artefacts due to applying acoustic inversion to elastic data of a model containing layer interfaces will be relatively minor. However, when diffractions are present, such as at fault boundaries and other strong lateral heterogeneities in the model, severe artefacts will be present. 


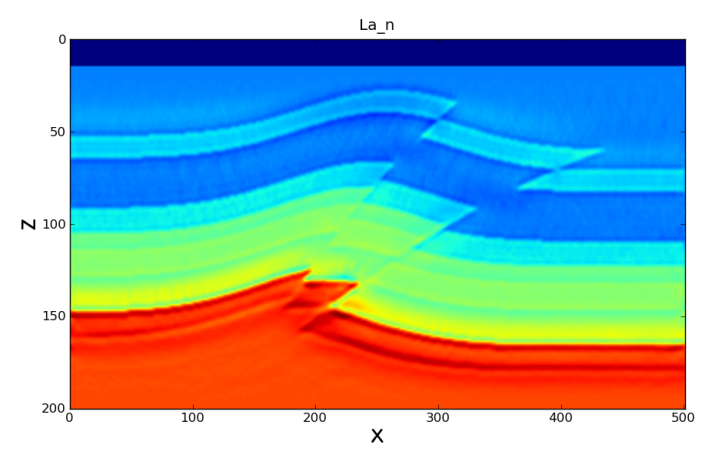

Figure 22. The result of an acoustic inversion of acoustic data in the $2 \mathrm{D}$ fault model showing the $P$-wave modulus $M=\lambda_{a}$ after 200 iterations. This result is of comparable quality to the result of elastic inversion of elastic data shown in Figure 11.

\section{CONCLUSIONS}

Several numerical experiments have been conducted to study the implications of applying acoustic invesion to elastic marine data. We found that it was necessary to strip the seafloor reflection from the simulation data in order for acoustic inversion to converge to a resonable quality subsurface image. In cases where the seafloor was not stripped, all effort of the inversion went to trying to match the seafloor reflection which was not possible. Namely, because the seismic waves entering the solid model underwent mode conversions, the seafloor reflection could never be matched by an acoustic simulation. However, by "stripping" the first seafloor reflection, it was possible to obtain convergence for the sub-seafloor layers. The stripping step means that first, the seafloor must be accurately modelled by an elastic simulation, and then subtracted from the data. Similarly, the acoustic simulations of the inversion must also

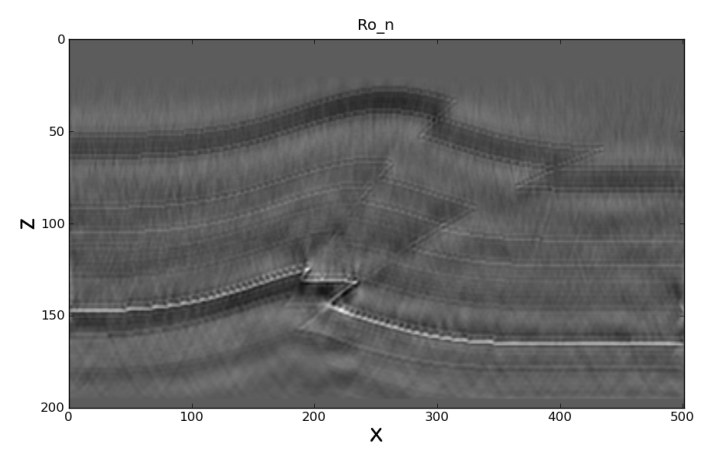

Figure 23. The acoustic inversion result for density $\rho$ of the acoustic marine data after 200 iterations. The true and starting models for density were both constant with $\rho=1$ and hence, are not shown here. 


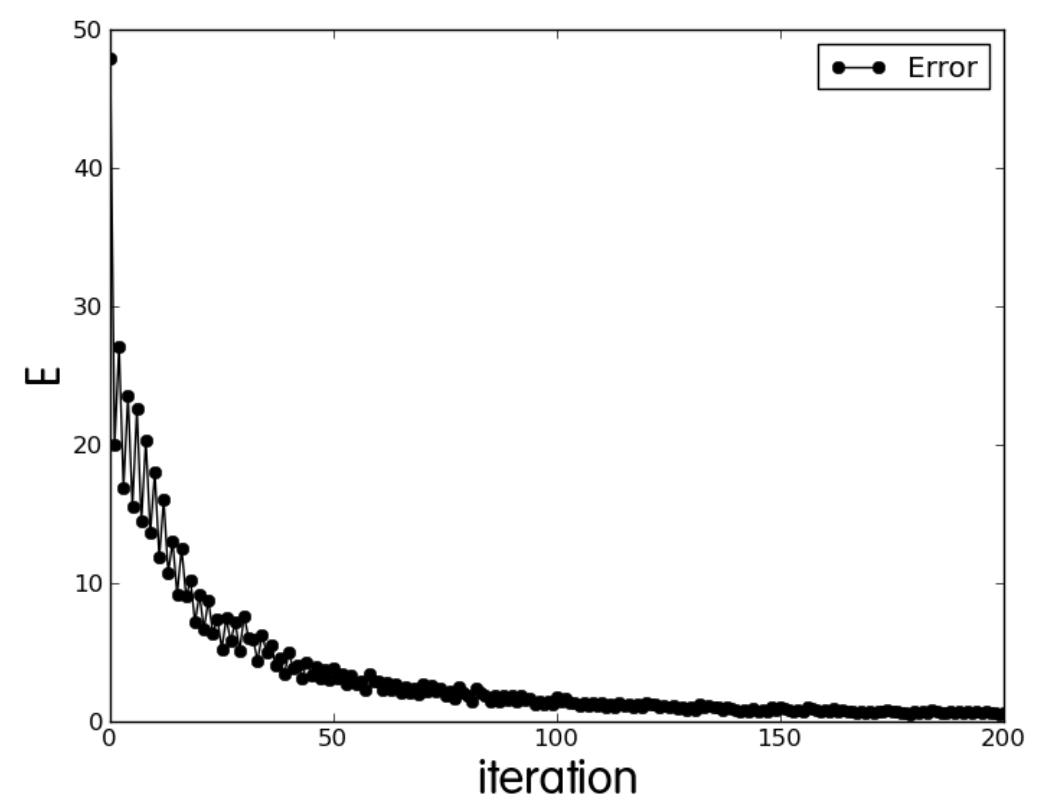

Figure 24. The error versus iteration for the acoustic inversion of acoustic marine data.

subtract the acoustic seafloor reflection from the acoustic simulated data. The following results apply to comparisons of an elastic inversion to a seafloor reflection stripped acoustic inversion of elastic data. it can be expected that clear and strong mode converted signals occur.

In the case of diffraction signals, an acoustic inversion of elastic data is likely to give very poor quality results consisting mainly of artefacts after several iterations, even for the case of a marine survey where an upper water layer is present. For the case of data consisting largely of reflections from layer interfaces, we have shown that acoustic inversion of elastic data of a marine survey can give adequate results for a single parameter inversion although a significant fraction of the data may be left unmatched corresponding to modes such as $P-S-S-P$ (ie. a $P$-wave in the water converting to a downgoing $S$-wave in the sub-seafloor solid layer, reflecting back as an $S$-wave to be converted back to a $P$-wave in the water layer). However, a much higher quality image, and of at least two parameters, $\lambda$ and $\mu$ or equivalent, can be obtained using fully elastic wavefield inversion, which matches the remaining data consisting of such mode converted sub-seafloor reflections.

In summary, for the case of large offset marine data in the case of both a hard and soft waterbottom, there is significant mode converted energy. This leads to artefacts in acoustic inversions due to the inversion attempting to fit the mode converted energy. These artefacts are minor for the case of layer reflections, and are severe for diffractions. In contrast, elastic 


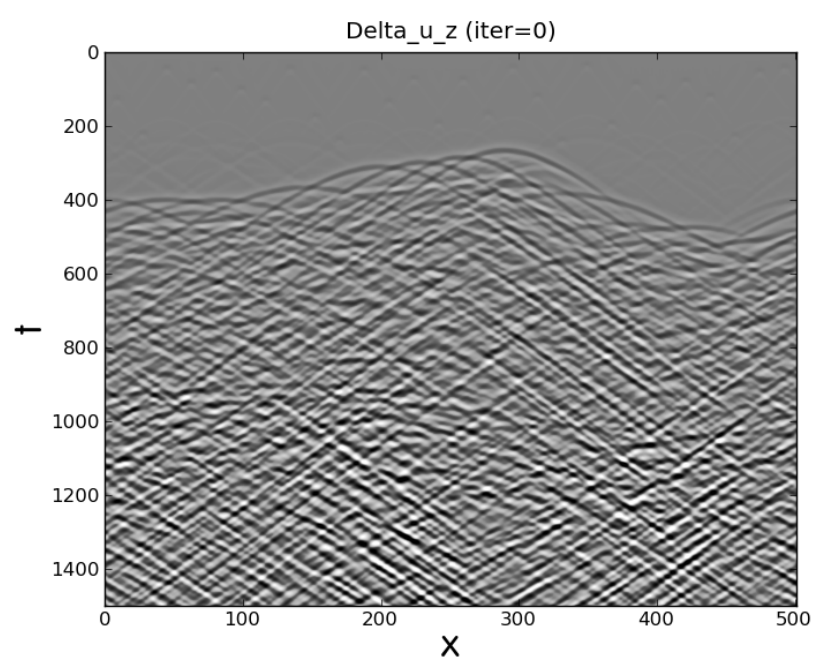

(a)

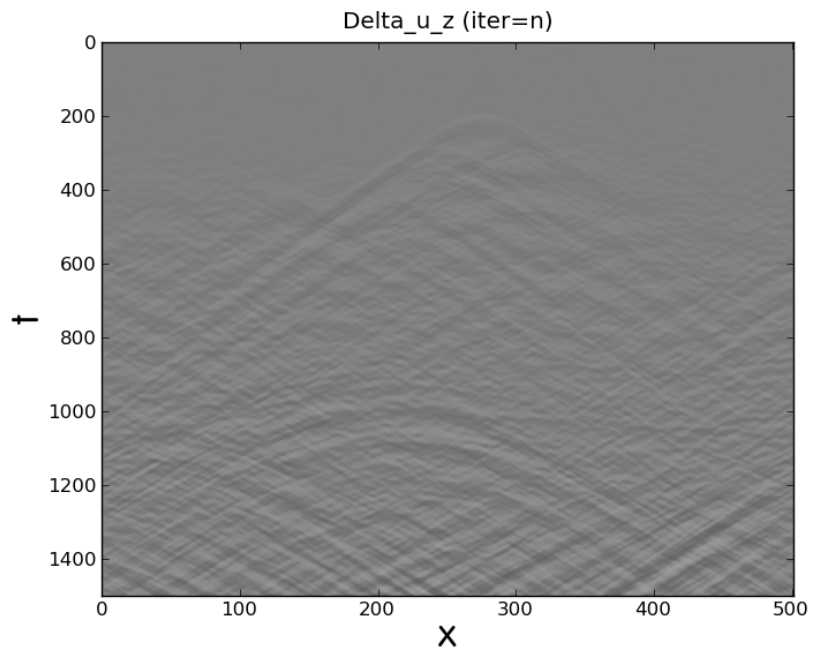

(b)

Figure 25. The initial residuals (a) and the final residuals after 200 iterations (b) for the acoustic inversion of acoustic marine data.

inversion leads to a clean image, and the resolution of two elastic parameters such as $\lambda$ and $\mu$ or $V_{p}$ and $V_{s}$.

Based on these results, we recommend that elastic inversion be carried out unless it has been shown for the specific survey, that acoustic inversion gives satisfactory results (ie. when artefacts are low enough in amplitude and when multi-parameter inversion is not beneficial), or that practical issues such as high attenuation of shear waves in soft sediments remove the benefits that may be obtained by elastic inversion, or when elastic computations are too expensive despite the benefits. These conclusions are based on idealized data without noise 


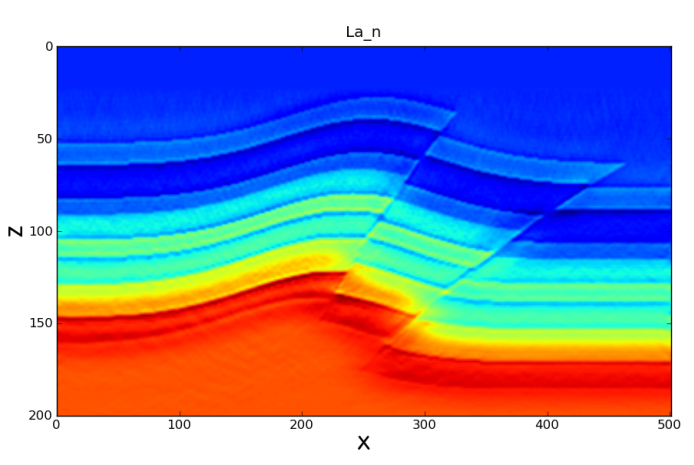

(a)

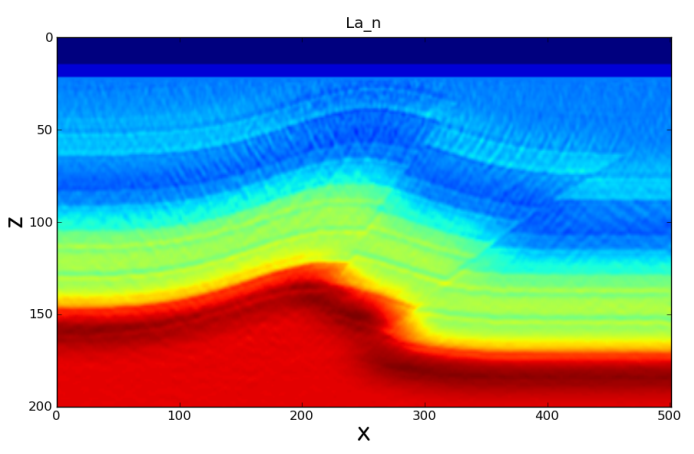

(c)

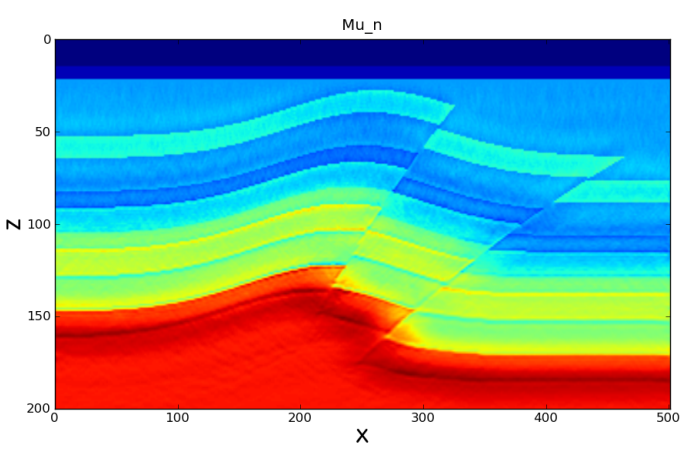

(b)

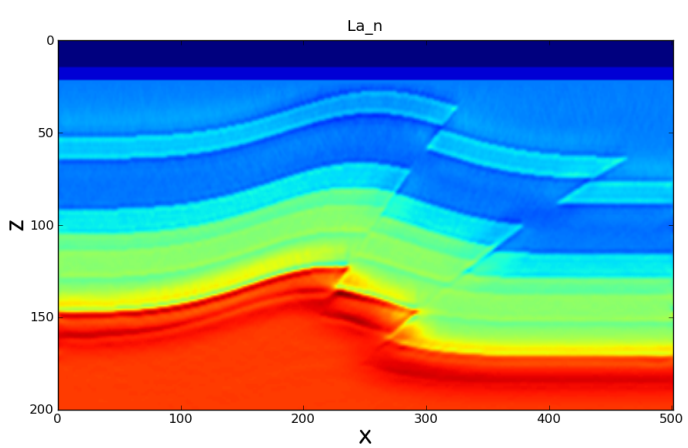

(d)

Figure 26. The inversion results after 200 iterations for the soft sediments case. Namely, the result for $\lambda(\mathrm{a})$ and $\mu$ (b) for the elastic inversion of elastic data, the result of acoustic inversion of elastic data (c), and the result for acoustic inversion of acoustic data (d).

for both the case of a hard and soft waterbottom. And we do not take into consideration issues that may arise in practice such as the possibility of high attenuation of shear waves in seafloor soft sediments. Nonetheless, the results suggest that one cannot assume an acoustic inversion is adequate due to it having matched most of the data as it may have matched the data by introducing artefacts into the solution, rather than having found the correct solution. However, if there are residuals left after an acoustic inversion corresponding to lower than $P$ wave velocity events, then it is possible or likely that these residuals originate from data due to mode conversions. In such cases, elastic inversion may be able to match these residuals, and in so doing, obtain a second image for the shear modulus $\mu$ or equivalent parameter such as $v_{s}$. As these results pertain to idealized theoretical data for the cases of a hard and soft waterbottom, subsequent research is required to validate these results for the practical constraints of real data, and to study how the conclusions of this study apply to more complex and realistic models.

In summary, the synthetic results show that acoustic inversion of large offset marine 
data leads to artefacts which are severe for diffractions and noticable but more minor for layer reflections. The artefacts are the result of the inversion attempting to match the mode converted energy at large offsets with the acoustic wave equation. In contrast, elastic inversion provides two clear images of the two elastic parameters, this being an improvement in both quality and quantity, for a relatively modest (less than about an order of magnitude) increase in computational cost.

\section{ACKNOWLEDGEMENTS}

We would like to thank John Etgen for reading the manuscript and helpful suggestions which greatly improved the quality of the paper. We also thank the two reviewers (Milena Marjanovic and anonymous) and the editor (Rene-Edouard Plessix) whose helpful comments lead to many improvements of the paper.

\section{APPENDIX A: NUMERICAL SIMULATION METHODOLOGY}

In the calculations in this paper, we solve the elastic wave-equation on a finite-difference mesh using a staggered grid formulation as described in Igel et al. (1995). The use of a staggered grid allows us to model pressure waves in an acoustic water layer using the same numerical scheme that is used for the elastic waves in the solid layers below the water bottom. Specifically, we need only set the second Lamé parameter $\mu=0$ in order to simulate an acoustic medium such as a water layer in which shear stresses are null and hence, shear waves do not propagate.

In this study, we define the grid spacing to be unity, and the Lamé parameters are also set to unity, or variations relative to unity. The use of such unitary parameters has no effect on interpretation of results which only depends on relative scales and dimensionless numbers such the ratio of offset relative to depth and ratio of the model width to model depth.

\section{A1 Elastic wave equation}

We require the ability to forward model seismic waves in a $2 \mathrm{D}$ medium. The equation for the conservation of linear momentum (ie. $F=m a$ ) in a continuum is given by

$$
\rho \ddot{u}_{i}=\frac{\partial \sigma_{i j}}{\partial x_{j}}+f_{i},
$$

where $\sigma_{i j}$ is the internal stress tensor in the medium, $u_{i}$ is the displacement, and $f_{i}$ is the body force source term. In an elastic medium, the stress tensor is related to the strain tensor 
$\epsilon_{i j}$ through the generalized Hooke's Law given by

$$
\sigma_{i j}=c_{i j k \ell} \epsilon_{k \ell}
$$

where the Einstein convention is used for repeated indices in the above equations (ie. repeated indices are summed). For the case of infinitesimal strains, the strain tensor is given by

$$
\epsilon_{i j}=\frac{1}{2}\left(\frac{\partial u_{i}}{\partial x_{j}}+\frac{\partial u_{j}}{\partial x_{i}}\right)
$$

For the case of an isotropic elastic medium, we have the following expression for the Hooke tensor (Aki \& Richards 2002)

$$
c_{i j k \ell}=\delta_{i j} \delta_{k \ell} \lambda+\left(\delta_{i k} \delta_{j \ell}+\delta_{i \ell} \delta_{j k}\right) \mu
$$

where $\delta_{i j}$ is the Kronecker delta, ie. $\delta_{i j}=1$ if $i=j$, and $\delta_{i j}=0$ if $i \neq j$. To propagate elastic waves in a $2 \mathrm{D}$ medium, one must first calculate the 3 independent components of the strain tensor using Equation (A.3), namely,

$$
\begin{aligned}
\epsilon_{00} & =\frac{\partial u_{0}}{\partial x_{0}}, \\
\epsilon_{11} & =\frac{\partial u_{1}}{\partial x_{1}}, \\
\epsilon_{01}=\epsilon_{10} & =\frac{1}{2}\left(\frac{\partial u_{0}}{\partial x_{1}}+\frac{\partial u_{1}}{\partial x_{0}}\right),
\end{aligned}
$$

where the indices 0 and 1 are respectively used to denote the $x$-axis and the $z$-axis. Subsequently, one must calculate the stress tensor $\sigma_{i j}$ using Equation (A.2) and (A.4), namely,

$$
\begin{aligned}
& \sigma_{00}=(\lambda+2 \mu) \epsilon_{00}+\lambda \epsilon_{11}, \\
& \sigma_{11}=(\lambda+2 \mu) \epsilon_{11}+\lambda \epsilon_{00}, \\
& \sigma_{01}=\sigma_{10}=\mu\left(\epsilon_{01}+\epsilon_{10}\right)=2 \mu \epsilon_{01} .
\end{aligned}
$$

We can then expand using Taylor series, the expression given by

$$
\begin{aligned}
\frac{u_{i}(t+\Delta t)-2 u_{i}(t)+u_{i}(t-\Delta t)}{\Delta t^{2}} & =\left(\frac{1}{\Delta t^{2}}\right)\left[\sum_{n=0}^{N}\left(\frac{(\Delta t)^{n}}{n !} \frac{\partial^{n} u_{i}}{\partial t^{n}}\right)-2 u_{i}(t)+\sum_{n=0}^{N}\left(\frac{(-\Delta t)^{n}}{n !} \frac{\partial^{n} u_{i}}{\partial t^{n}}\right)\right] \\
& =\left(\frac{2}{\Delta t^{2}}\right) \sum_{m=1}^{M}\left(\frac{\Delta t^{(2 m)}}{(2 m) !} \frac{\partial^{(2 m)} u_{i}}{\partial t^{(2 m)}}\right) .
\end{aligned}
$$

One can then rearrange Equation (A.7) to obtain the explicit finite-difference scheme to order $2 M$ given by

$$
u_{i}(t+\Delta t)=2 u_{i}(t)-u_{i}(t-\Delta t)+2 \sum_{m=1}^{M}\left(\frac{\Delta t^{(2 m)}}{(2 m) !} \frac{\partial^{(2 m)} u_{i}}{\partial t^{(2 m)}}\right)
$$


where the $(2 m)$-th time derivative of the wavefield $u_{i}$ can be calculated by applying the wave-equation given by Equation (A.1) $m$ times. Namely, one first calculates the strains $\varepsilon_{i j}$ from the displacements using Equation (A.3). Subsequently, one applies Hooke's Law given by Equation (A.2) to compute the stress tensor $\sigma_{i j}$. Finally, one can calculate the second time derivative of the displacement via the wave equation given by Equation (A.1).

To calculate the $(2 m)$-th time derivative of the displacement, one repeats this sequence $(m-1)$ additional times. Namely, one feeds the second time derivative of the displacement into the strain-displacement relation given by Equation (A.3) to compute the second time derivative of the strains. Then, application of Hooke's Law given by Equation (A.2) yields the second time derivative of the stress tensor $\ddot{\sigma}_{i j}$. Finally, one can calculate the 4 -th time derivative of displacement by applying the wave-equation given by Equation (A.1) to the second time derivative of the stress tensor $\ddot{\sigma}_{i j}$. Hence, the even time derivatives to any order can be computed to the precision of the spatial derivative operator. If we take this series to second order, one obtains the second order explicit finite-difference extrapolation equation given by

$$
\begin{aligned}
& u_{0}(t+\Delta t)=2 u_{0}(t)-u_{0}(t-\Delta t)+\frac{\Delta t^{2}}{\rho}\left(\frac{\partial \sigma_{00}}{\partial x_{0}}+\frac{\partial \sigma_{01}}{\partial x_{1}}\right), \\
& u_{1}(t+\Delta t)=2 u_{1}(t)-u_{1}(t-\Delta t)+\frac{\Delta t^{2}}{\rho}\left(\frac{\partial \sigma_{11}}{\partial x_{1}}+\frac{\partial \sigma_{01}}{\partial x_{0}}\right) .
\end{aligned}
$$

In this paper, we use a 12-point convolutional operator to compute space derivatives (Mora 1986; Igel 2017) and we calculate the time derivatives to second order (ie. $M=1$ in Equation (A.8)). Namely, the spatial derivatives on the staggered grid are given by the pseudospectral operator $i k_{x} \exp \left(i k_{x} \Delta x / 2\right)$ multiplied by a Gaussian function (see Mora (1986); Igel et al. (1995)). For efficiency, these convolutional spatial derivatives are computed by Fourier transforming the displacement wavefields, followed by multiplication by the staggered derivative operator and inverse Fourier transforming the result, where the Fourier transforms required by this pseudo-spectral spatial derivative operator are calculated using the parallel fftw library called from a python source code (see https://pypi.python.org/pypi/pyFFTW for fftw and www.python.org for python). All calculations were done on a small home workstation, and these limited computational resources led to restrictions of the size of the calculations to 2 D finite-difference meshes not larger than around $502 \times 232$ units. 


\section{APPENDIX B: INVERSION FORMULATION}

The inverse problem is expressed in terms of finding the most probable model $\mathbf{m}$ for a given set of data observations $\mathbf{d}_{0}$ (eg. Menke (2012); Tarantola (2005)). Consider the Gaussian probability density function

$$
P \propto \exp \left[-\frac{1}{2}\left(\Delta \mathbf{d}^{T} \mathbf{C}_{\mathbf{d}}^{-\mathbf{1}} \Delta \mathbf{d}+\Delta \mathbf{m}^{T} \mathbf{C}_{\mathbf{m}}^{-\mathbf{1}} \Delta \mathbf{m}\right)\right]=\exp (-E),
$$

where $\mathbf{C}_{\mathbf{d}}$ is the data covariance matrix, $\mathbf{C}_{\mathbf{m}}$ is the model covarience matrix, and

$$
\Delta \mathbf{d}=\mathbf{d}(\mathbf{m})-\mathbf{d}_{0} \quad, \quad \Delta \mathbf{m}=\mathbf{m}-\mathbf{m}_{0}
$$

are respectively the data mismatch or residual defined as the difference between the calculated data $\mathbf{d}(\mathbf{m})$ and observed data $\mathbf{d}_{0}$, and the model mismatch between the model $\mathbf{m}$ and an a priori model denoted $\mathbf{m}_{0}$. The most probable or maximum likelihood solution, when the probability $P$ is maximal, occurs when the derivative of the square error functional

$$
E=\frac{1}{2}\left(\Delta \mathbf{d}^{T} \mathbf{C}_{\mathbf{d}}^{-\mathbf{1}} \Delta \mathbf{d}+\Delta \mathbf{m}^{T} \mathbf{C}_{\mathbf{m}}^{-\mathbf{1}} \Delta \mathbf{m}\right),
$$

is minimized. Hence, we seek a solution when the derivative of the error functional or gradient vector $\mathbf{g}=\partial E / \partial \mathbf{m}^{T}=0$. Namely, we seek the solution to

$$
\mathbf{g}=\frac{\partial E}{\partial \mathbf{m}^{T}}=\mathbf{D}^{T} \mathbf{C}_{\mathbf{d}}^{-\mathbf{1}} \Delta \mathbf{d}+\mathbf{C}_{\mathbf{m}}^{-\mathbf{1}} \Delta \mathbf{m}=0
$$

where $\mathbf{D}=\partial \mathbf{d} / \partial \mathbf{m}$.

In the following, for simplicity in the subsequent idealized inversions, we assume that the model parameters are unconstrained so $\mathbf{C}_{\mathbf{m}}^{-1}=0$, and the data errors are uncorrelated and constant so $\mathbf{C}_{\mathbf{d}}^{-1}=\mathbf{I} / \sigma_{d}^{2}$. In this case, we need to solve

$$
\mathbf{g}=\mathbf{D}^{T} \Delta \mathbf{d}=0
$$

This can achieved by a conjugate gradient algorithm, provided the gradient can be calculated.

For isotropic elastic media, the equations for the gradient are given by (see Mora (1987b); Tarantola (1988))

$$
\begin{aligned}
& \delta \hat{\rho}=\sum_{S} \int d t \frac{\partial u_{i}}{\partial t} \frac{\partial \psi_{i}}{\partial t}=\sum_{S} \int d t \dot{u}_{i} \dot{\psi}_{i}, \\
& \delta \hat{\lambda}=-\sum_{S} \int d t \frac{\partial u_{i}}{\partial x_{i}} \frac{\partial \psi_{j}}{\partial x_{j}}=-\sum_{S} \int d t\left(\nabla_{i} u_{i}\right)\left(\nabla_{j} u_{j}\right)=-\sum_{S} \int d t \epsilon_{i i} \epsilon_{j j}^{\dagger}, \\
& \delta \hat{\mu}=-\sum_{S} \int d t \frac{1}{\sqrt{2}}\left(\frac{\partial u_{i}}{\partial x_{j}}+\frac{\partial u_{j}}{\partial x_{i}}\right) \frac{1}{\sqrt{2}}\left(\frac{\partial \psi_{i}}{\partial x_{j}}+\frac{\partial \psi_{j}}{\partial x_{i}}\right)=-\sum_{S} \int d t 2 \epsilon_{i j} \epsilon_{i j}^{\dagger},
\end{aligned}
$$

where the wavefield $\psi_{i}(x, t)$ is the back propagated data residuals, $\epsilon_{i j}$ is denotes the strain 
tensor of wavefield $u_{i}, \epsilon_{i j}^{\dagger}$ is denotes the strain tensor of the adjoint wavefield $\psi_{i}$, and the Einstein convention is used for repeated indices (ie. repeated indices are summed). Note that the equations for the general case in terms of the density $\rho$, the Hooke tensor coefficients $c_{i j k \ell}$, and the attenuation are provided by Tarantola (1988). In the following, we denote the gradient vector at the $n$-th iteration as $\mathbf{g}_{n}$ where

$$
\mathbf{g}=[\delta \hat{\rho}, \delta \hat{\lambda}, \delta \hat{\mu}]^{T}
$$

\section{B1 Conjugate gradient method}

The conjugate gradient method involves iterations updating the model in the conjugate gradient direction $\mathbf{c}_{n}$ as follows

$$
\mathbf{m}_{n+1}=\mathbf{m}_{n}-\alpha_{n} \mathbf{c}_{n}
$$

where $\alpha_{n}$ is a scalar step-length and $\mathbf{c}_{n}$ is used to denote the conjugate direction at the $n$-th iteration which is calculated as a linear combination of the current gradient vector and the last conjugate direction. Namely,

$$
\mathbf{c}_{n}=\mathbf{g}_{n}+\beta_{n} \mathbf{c}_{n-1}
$$

where $\beta_{n}$ is a scalar which can be calculated according to a number of different formulas which will be specified in the following. The benefit of the conjugate gradient method is that it avoids the zig-zag behaviour sufferred by the steepest descent method as it progresses down a banana shaped objective function. The steplength $\alpha_{n}$ can be calculated by a line search, with an initial estimate of $\alpha_{n}$ calculated using the following formula

$$
\alpha=\frac{\mathbf{c}^{T} \mathbf{c}}{\mathbf{c}^{T} \mathbf{D}^{T} \mathbf{C}_{\mathbf{d}}^{-1} \mathbf{D} \mathbf{c}+\mathbf{c}^{T} \mathbf{C}_{\mathbf{m}}^{-1} \mathbf{c}},
$$

which can be derived by solving $\partial \varepsilon / \partial \alpha_{n}=0$ (see Mora (1987a)).

The conjugate gradient method starts by an iteration in the direction of steepest descent (ie. $\beta_{1}=0$ ) and subsequently, iterations are calculated according to Equation (B.9). After the first iteration, the value of the scalar $\beta_{n}$ can be calculated by various formulas which are named after their developers. Four popular choices are the Fletcher-Reeves, Polak-Ribiére, Hestenes-Steifl, and Dau-Yuan methods (denoted as FR, PR, HS and DY). Each of these formulas yield the same result for quadratic objective functions (linear theories), and result in the solution after exactly $n$ iterations where $n$ is the number of unknowns or model parameters being solved for. However, the performance of these different methods to calculate $\beta_{n}$ varies. We have tested each of these popular choices for the examples of Full Wavefield Inversion 
shown in this paper, and found that for the cases studied, that the performance (in order of descending performance) is $P R, F R, D Y$ and lastly, $H S$. The $P R$ and $F R$ methods were similar in performance with $P R$ being slightly better, and these were both significantly better in performance than the $D Y$ and $S H$ methods which were also similar in performance. For this reason, we use the Polak-Ribiére method given by

$$
\beta_{n}^{P R}=\frac{\mathbf{g}_{n}^{T}\left(\mathbf{g}_{n}-\mathbf{g}_{n-1}\right)}{\mathbf{g}_{n-1}^{T} \mathbf{g}_{n-1}}
$$

At some stage, progress using the conjugate gradient method may slow or stop due to loss of conjugacy for nonlinear functions (ie. non-quadratic objective functions). If this occurs, a reset to the direction of steepest descent will allow subsequent iterations to regain conjugacy. A popular choice for $\beta$ that provides an automatic direction reset and, provides the best performance for the examples in this paper is given by

$$
\beta=\max \left\{0, \beta^{P R}\right\} .
$$

\section{REFERENCES}

Agudo, O. C., da Silva, N., Warner, M., \& Morgan, J., 2016. Acoustic full-waveform inversion in an elastic world, in 86th Ann. Internat. Mtg, pp. 1058-1062, Soc. of Expl. Geophys.

Aki, K. \& Richards, P. G., 2002. Quantitative Seismology: Theory and Methods, University Science Books, 2nd edn.

Albertin, U., Shen, P., Sekar, A., Johnsen, T., Wu, C., Nihei, K., \& Bube, K., 2016. 3d orthorhombic elastic full-waveform inversion in the reflection domain from hydrophone data, in 86th Ann. Internat. Mtg, pp. 1094-1098, Soc. of Expl. Geophys.

Barnes, C. \& Charara, M., 2009. The domain of applicability of acoustic full-waveform inversion for marine seismic data, GEOPHYSICS, 74(6), WCC91-WCC103.

Chapman, C. H., Hobro, J. W. D., \& Robertsson, J. O. A., 2014. Correcting an acoustic wavefield for elastic effects, Geophysical Journal International, 197(2), 1196-1214.

He, W. \& Plessix, R., 2016. Analysis of different parameterisations of waveform inversion of compressional body waves in an elastic transverse isotropic earth with a vertical axis of symmetry, Geophysical Prospecting, 65(4), 1004-1024.

He, W., Plessix, R.-E., \& Singh, S., 2018. Parametrization study of the land multiparameter vti elastic waveform inversion, Geophysical Journal International, 213(3), 1660-1672.

Hobro, J. W. D., Chapman, C. H., \& Robertsson, J. O. A., 2014. A method for correcting acoustic finite-difference amplitudes for elastic effects, GEOPHYSICS, 79(4), T243-T255.

Igel, H., 2017. Computational Seismology, Oxford University Press, Oxford. 
Igel, H., Mora, P., \& Riollet, B., 1995. Anisotropic wave propagation through finitedifference grids, GEOPHYSICS, 60(4), 1203-1216.

Lailly, P., 1983. The seismic inverse problem as a sequence of before stack migrations, in Conference on inverse scattering: theory and application, pp. 206-220.

Marelli, S., Maurer, H., \& Manukyan, E., 2012. Validity of the acoustic approximation in full-waveform seismic crosshole tomography, GEOPHYSICS, 77(3), R129-R139.

Menke, W., 2012. Geophysical data analysis: Discrete inverse theory:MATLAB edition, vol. 45, Academic press.

Mora, P., 1986. Elastic finite-differences with convolutional operators, Stanford Exploration Project, Tech. Rep. 48, $272-289$.

Mora, P., 1987a. Elastic wavefield inversion, Ph.D. Thesis, Stanford University (also published as SEP-52, Stanford Exploration Project technical report \# 52).

Mora, P., 1987b. Nonlinear twodimensional elastic inversion of multioffset seismic data, GEOPHYSICS, 52(9), 1211-1228.

Mulder, W. \& Plessix, R., 2008. Exploring some issues in acoustic full waveform inversion, Geophysical Prospecting, 56(6), 827-841.

Schiemenz, A. \& Igel, H., 2013. Accelerated 3-d full-waveform inversion using simultaneously encoded sources in the time domain: application to valhall ocean-bottom cable data, Geophysical Journal International, 195(3), 1970.

Shipp, R. M. \& Singh, S. C., 2002. Two-dimensional full wavefield inversion of wide-aperture marine seismic streamer data, Geophysical Journal International, 151(2), 325-344.

Tarantola, A., 1984. Inversion of seismic reflection data in the acoustic approximation, GEOPHYSICS, 49(8), 1259-1266.

Tarantola, A., 1988. Theoretical background for the inversion of seismic waveforms, including elasticity and attenuation, in Scattering and Attenuations of Seismic Waves, Part I, pp. 365-399, Birkhäuser Basel.

Tarantola, A., 2005. Inverse problem theory and methods for model parameter estimation, SIAM.

Vigh, D., Starr, E. W., \& Elapavuluri, P., 2009. Acoustic waveform inversion vs. elastic data., in 79th Ann. Internat. Mtg, pp. 2298-2301, Soc. of Expl. Geophys.

Willemsen, B., Cao, J., \& Roy, B., 2016. The impact of the acoustic approximation on time-lapse fwi, in 86th Ann. Internat. Mtg, pp. 5435-5440, Soc. of Expl. Geophys. 